\title{
Discretization of Expression Quantitative Trait Loci in Association Analysis Between Genotypes and Expression Data
}

\author{
Andrés R. Masegosa ${ }^{1}$, Rubén Armañanzas ${ }^{2}$, María M. Abad-Grau ${ }^{*}$, , Víctor Potenciano ${ }^{3}$, \\ Serafín Moral ${ }^{1}$, Pedro Larrañaga ${ }^{4}$, Concha Bielza ${ }^{4}$ and Fuencisla Matesanz ${ }^{5}$ \\ ${ }^{1}$ CITIC, Universidad de Granada, Granada, Spain \\ ${ }^{2}$ Krasnow Institute for Advanced Study, George Mason University, Fairfax, VA, USA \\ ${ }^{3}$ Poten Dynamics, Granada, Spain \\ ${ }^{4}$ Computational Intelligence Group, Departamento de Inteligencia Artificial, Universidad Politécnica \\ de Madrid, Madrid, Spain \\ ${ }^{5}$ Instituto de Parasitología Lopez Neyra, CSIC, Granada, Spain
}

\begin{abstract}
Expression quantitative trait loct are used as a tool to identify genetic causes of natural vartation in gene expression. Only in a few cases the expression of a gene is controlled by a variant on a single genetic marker. There is a plethora of different complexity levels of Interaction effects within markers, within genes and between marker and genes. This complex lty challenges blostatistictans and bloinformatitians every day and makes findings difflcult to appear. As a way to simplify analysis and better control confounders, we trled a new approach for association analysis between genotypes and expression data. We pursued to understand whether discretization of expression data can be useful in genome-transcrptome assoctation analyses. By discretizing the dependent varlable, algorlthms for learning classifters from data as well as performing block selectlon were used to help understanding the relationship between the expression of a gene and genetic markers. We present the results of using this approach to detect new possible causes of expression vartation of DRB5, a gene playing an important role within the immune system. Together with expression of gene DRB5 obtained from the classical microarray technology, we have also measured DRB5 expression by using the more recent next-generation sequencing technology. A supplementary website including a link to the software with the method implemented can be found at http: //blos.ugr.es/DRB5.
\end{abstract}

\section{INTRODUCTION}

Assoclation between genotypes and mRNA transcript levels may help elucidating genetic basis of complex diseases by analyzing whenever genetic varlants affect gene expression. Therefore, a genetic variant affecting a disease may be found also in association with the expression level of a gene. However, it is not straightforward to understand whether it may truly alter gene transcription or splicing, 1.e. It may be an expression quantitative tralt loct (eQTL), or just being in linkage disequilibrium (LD) with the real cause [1]. Moreover, because of small sample sizes and limited computational resources, regression models using several input varlables have given results hardly reproduclble and most successful association analyses only succeeded when testing a single polymorphic locus (SNP) against the

*Address correspondence to this author at the Departamento Lenguajes $y$ Sistemas Informáticos, C/ Periodista Daniel Saucedo Aranda s/n, Granada 18071. Spain; Tel: +34 958240634; Fax: +34 958243179; E-mail: mabad@ugr.es

${ }^{5}$ This work is an extension of the following conference paper: Andres $R$ Masegosa, María del Mar Abad-Grau, Serafín Moral, and Fuencisla Matesanz. Learning classif iers from discretized expression quantltatl ve traitloci. IWBBIO, page 427-436. Copicentro Ed itorial, (2013) expression of a gene instead of considering more than one SNP at a time [1-3]. We have used a different approach to measure assoclation between SNPs and gene expression data which relles on a pre-discretization of expression data as a way to simpllfy input data and improve performance compared with standard regression models. Discretization of gene expression data is commonly performed when they are used as the input variables to predict different phenotypes such as cellular classification in cancer [4-6]. With this simplification, we are able to use data to learn a classifter, 1.e. a model that relates how input varlables, the SNPs, and their Interactions, affect a discrete output varlable, with values interpreted as high and low gene expression if $\mathrm{it}$ is the case of only two blns or high, regular and low gene expression if it is the case of three bins. By using classiflers Instead of regression functions, other more complex analyses can be made, such as considering multiple SNPs at a time or haplotyping analysis [7]; it could be reduced the computational and statistical complexity; and, In consequence, to have a more affordable alternatlve. B ut most Important, a different approach may help shed light about the main features of the data analyzed and thus about different interaction patterns between genes and regulatory proteins affecting their expression and about their assoclation with SNPs within a block of high LD. Moreover, 
the use of different classifiers under different assumptions and the use of different learning algorithms under different approaches may help to increase chances of discovering new regulation patterns.

We focused on gene HLA-DRB 5 (DRB5). We chose this gene because the expression pattern in the first two data sets (described at Section 2.8) analyzed -those obtained by using microarray technology- showed two non-overlapping distrlbutions of low and high expression levels (see Fig. 1) and it was easily translated to a binary variable.

DRB 5 is one of the genes that encode $\beta$ chains for the DR HLA class II receptor. The HLA genes are located on the short arm of chromosome 6 and are organized in three reglons: MHC class I, MHC class II and MHC class III. HLA class II genes encode glycoprotelns expressed primarily on antigen-presenting cells where they present processed antigenic peptides to CD4+ T cells. The DR $\beta$ chain is encoded by 4 gens DRBI, 3, 4, and 5 . There are also other pseudogenes that do not produce a proteln: DRB 2, 7, 8, and 9. Not everybody has a copy of each gene or pseudogene. There are 5 different haplotypes with different combinations of genes. DRB5 is only present in DR51 haplotype. This haplotype has been associated with immune related diseases susceptibility. In particular, the DRB5*0101- DRBI*1501-DQAl*0102- DQB1*0602 haplotype has been assoclated with Multiple Sclerosis (MS) In the North European population [8]. The strong linkage disequilibrlum among the varlants that integrate the mentioned haplotype, in the Caucasian population, makes very difficult to determine the primary associated varlant The polymorphisms at the DRB genes conferred different propertles for antigen presentation and this has been postulated as the pathogenic mechanism. However, it could be not the only explanation for the HLA Class II association with Multiple Sclerosis. It has been described polymorphisms that alter HLA gene expression assoclated with Multiple Sclerosis susceptlbillty [9-11], which open the question of the role of the DRB gene expression levels in the pathology. In fact the abllity to induce actlve experimental autolmmune encephalomyelitts (EAE), an animal model for

DRB5-Mlcroamay8-CEU

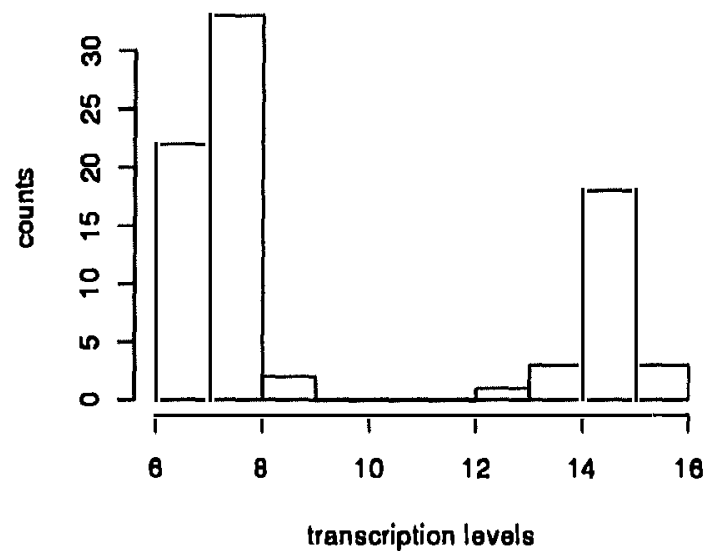

MS disease, was increased in animals expressing higher levels of DRB5*01: 01, pointing to a role of the levels of expression of this gene in susceptlbility [12].

The rest of the paper is divided in three main sections. In Section 2 we describe the proposed methods and the employed data sets. Results appear in Section 3 and a discussion can be read in Section 4.

\section{MATERIALS AND METHODS}

In Section 2.1, we start glving a motivation and a rough description of our approach. Section 2.2 contains the detalls of our discretization algorithm. Sections 2.3 and 2.4 present the basics of classification and regression models, respectlvely. Section 2.5 explains how these two different models can be compared between them. Section 2.6 detalls a preprocessing step for grouping SNPs. We show a flowchart in Section 2.7 with the steps followed by our proposed method. Finally in Section 2.8 we describe the data sets we used and the procedures we followed to obtain them.

\subsection{Motivation}

Our discretization step of mRNA transcript levels is empirically motlvated. Partlcularly, it arose when we observed the histograms of the expression level of the gene DRB5 measured by using microarrays from the RNA of lymphoblastold cell lines in two different populations (one from Utah, USA composed of individuals with ancestries in Northern and Western Europe (CEU), and the other (YRI) with Individuals from Yoruba, Nigeria. These histograms are shown in Fig. (1).

As it can be seen, two different groups, within each population, neatly arose: one group with relative low expression levels and another group with relative high expression levels.

In this paper, we bulld on the identification of these two groups (1.e. under-expressed and over-expressed) to measure SNP-expresslon level assoclations. Our approach to measure

\section{DRB5-Mlcroamays-YRI}

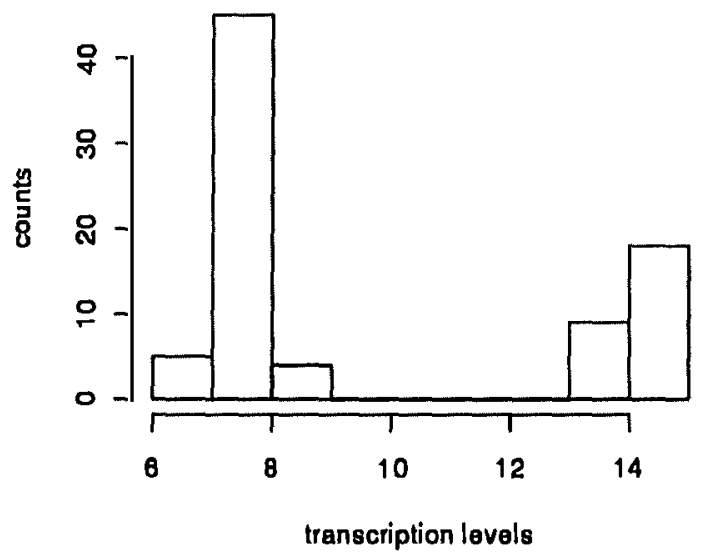

Fig. (1). Count-histograms of transcription levels ( $x$-axis) for DRB5 in CEU-array data. set (a) and YRI-array data set (b). 
these associations consists on employing statistical methods that considers the expression level as a blnary varlable (ternarles varlables were later on explored in this work). This approach can be seen as an alternative, but not unique, methodology to those prevlously proposed statistical approaches which treat the expression level of a gene as a continuous real value [1-3] (e.g. the employment of a correlation test such as Spearman to measure the association between a single SNP and the expression of a given gene). More formally, we consider the following causal statistica models to explain the influence of one SNP over the expression of a target gene, the DRB5 gene in this work. These models are graphically described in Fig. (2) following the notation employed in Bayesian networks [13]. Under this notation, random varlables are represented by round nodes and direct causal statistical influences are represented by directed edges. In this figure, the random variable $S$ models the different values that a given SNP takes in a given population. In our case, we assume that one SNP can take three different values: 0,1 and 2. Similarly, the random variable $G$ models the expression level of any target gene in the population, which is assumed to be a random continuous value. Many previous approaches [1-3] for measuring SNP expression levels associations implicitly employ the "ContInuous Model": they test whether one SNP directly influences or not the continuous expression level of the target gene. In this paper we advocate for the "Discretization Model". This model assumes the existence of another hidden discrete varlable, denoted by $\mathrm{H}$ in $\mathrm{Fig}$. (2). This varlable would represent a non-observable blological mechanism which is modulated by some SNP and, in turn, triggers the expression level, low or high, of the target gene. In result, we say that when one SNP regulates the expression level of a gene, it is not a direct cause of this regulation because it firstly affects this non-observable blological mechanism denoted by $\mathrm{H}$. This mechanism would be the direct cause of the particular expression of the gene.

The assumption of the above model leaded us to use the alternative methods for measuring the SNP-expression levels assoclations presented in this work. We assume we have a data set with $\mathrm{M}$ members of a given population and for each member in our data set we can measure the value of a given set of SNPs, which are denoted by $S=\left\{S_{1}, \ldots, S_{N}\right\}$. Under these settings, $s_{13}$ will denote the value of the SNP Si for the $j$-th individual in the data set, with $f \in\{1, \ldots, N\}$ and $j \in\{1$, .... M . Additionally, we also have measures for the expression level of a target gene $G$ for each member of the data set. The expression level of the $j$-th member of the data set will be denoted by $g_{j}$ with $\mathbf{j} \in\{1, \ldots, M\}$ and the range of values that $G$ can take will be denoted by $\operatorname{Val}(G)$. The set of expression values for the whole data set will be denoted by g. Our goal is to measure the association level between a given SNP Si and the expression of the gene $G$, assuming the "Discretization Model". We carried out this evaluation performing the two following steps:

Step 1: In this step we inferred, for each member of the data set, the values of the hidden varlable $H$, denoted by $h_{j}$ with $j \in\{1, \ldots, M\}$. We will also denote by $h$ to the whole set of $h_{1}$ values. In that way, we evaluated whether the expression level of the $\mathbf{j}$-th member of the data set is low or high. In the case of data sets with count-histograms such as the one shown in Fig. (1), it is straightforward to infer these values: if $g_{j}<10$ then $h_{j}=$ low, otherwise $h_{j}=$ high (the cutoff polnt could be any value between 9 and 12). For data sets where the two groups overlap, and for the cases in which there are reasons to consider more than two groups, we present in Section 2.2 an automatic approach based on the EM algorithm [14] and the Gaussian mixture model [15] to infer the hyvalues using the expression level values $g_{j}$.

Step 2: Once we computed the values of the varlable $\mathbf{H}$ for the data set from the population under study (i.e. h), we measured the assoclation between SNPs and gene expression by using the varlable $H$ instead of the varlable $G$. Because the varlable $H$ is discrete, a different family of statistical approaches became avallable for this purpose. In Section 2.3, we detall our proposal to accomplish this step.

\subsection{The EM Algorithm and the Gaussian Mixture Model (Step 1)}

In this section we glve detalls about the "Step 1" of our proposal, as detalled in the previous sectlon. The goal of this step was to infer the set of values $h$ using the values of the gene expression $\mathrm{g}$. For this purpose, we assumed that the gene expression level followed a Gaussian mixture model (GMM) [15]. Under this model the expression level of a gene is normally distributed conditioned to $\mathrm{H}: \mathrm{P}(\mathrm{G} \mid \mathrm{H}=\mathrm{h}) \sim$ Gaussian $\left(\mu_{h}, \sigma_{h}\right)$, where $\mu_{h}$ and $\sigma_{h}$ denote the mean and the standard deviation of the Gaussian distribution when $\mathrm{H}=\mathrm{h}_{\text {; }}$ and, then, the distribution of $G$ follows a weighted mixture

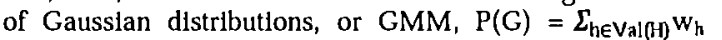
Gausslan $\left(\mu_{h}, \sigma_{h}\right)$, where $w_{h}$ is the welght of the $h$-th component of the mixture, $w_{h}=P(H=h)$. Although the varlable $\mathrm{H}$ is not observed, we can employ the EM algorithm [14] to infer the parameters which define this mixture: $w=$ $\left\{w_{1}, \ldots, w_{K}\right\}, \mu=\left\{\mu_{1}, \ldots, \mu_{K}\right\}$ and $\sigma=\left\{\sigma_{1}, \ldots, \sigma_{K}\right\}$, where $K$ denotes the number of values of $\mathrm{H}$. Once these parameters are estimated with the EM algorithm, the $h_{j}$ values were computed as follows:

$$
\begin{aligned}
h_{j}=\underset{h \in \operatorname{Val}(H)}{\operatorname{argmax}} P\left(g_{j} \mid H=h\right) P(H=h) \\
=\underset{h \in \operatorname{Val}(H)}{\operatorname{argmax}} \frac{w_{h}}{\sigma_{h} \sqrt{2 \pi}} e^{\frac{-\left(g_{j}-\mu_{h}\right)^{2}}{2 \sigma_{h}^{2}}}
\end{aligned}
$$
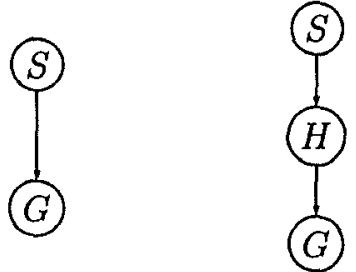

Continuous Model Discretization Model

Fig. (2). The two causal statistical models evaluated in this work. Random varlables are represented by round nodes and direct causal statistical influences are represented by directed edges. $S$ models the different values that a given SNP takes in a glven population; $G$ models the expression level of any target gene, which is assumed to be a random contlnuous value. $H$ is a hidden varlable which models a non-observable blological mechantsm which is modulated by some SNP and, in turn, trlggers the expression level $G$ of the target gene 


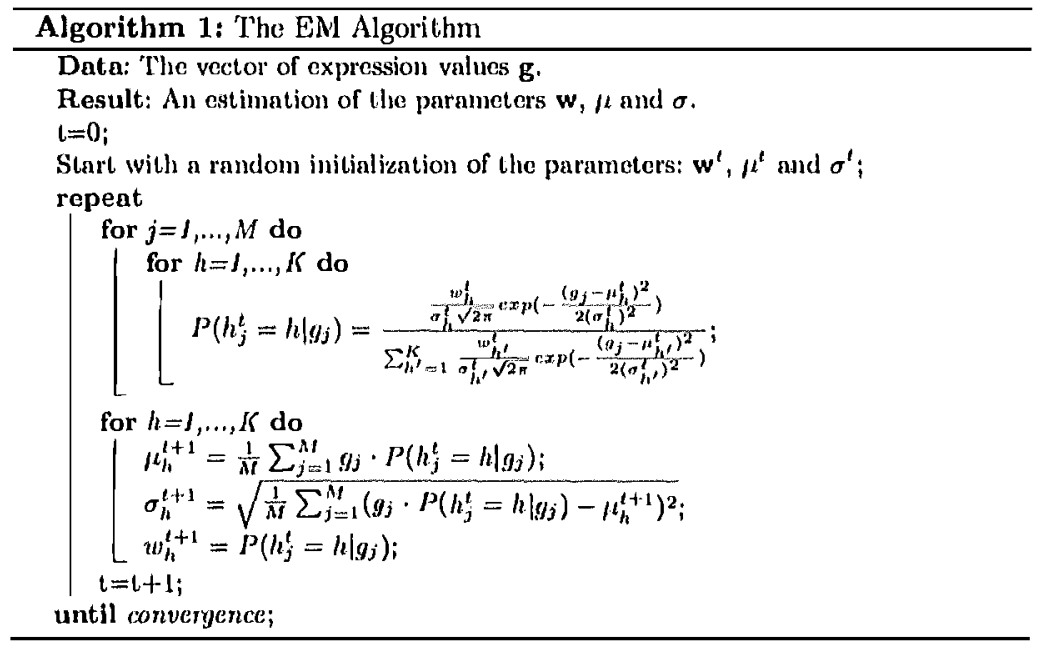

In Algorithm 1 we give a pseudo-code description of the EM algorithm. This algorlthm starts with a random initialization of the parameters $w, \mu$ and $\sigma$ (when initializing $w$, it must be satisfied that $\sum_{h} w_{h}=1$ ). The algorithm iterates untll convergence (1.e., when the parameters in the teration $t$ +1 are equal to the parameters in iteration $t)$. However, it is well known that different executions of the algorithm can lead to different estimations of the parameters, where each different estimationcorresponds to alternative local maximum of the likellhood function. To avold low quality convergence points, the EM algorlthm was run 100 times as done and the solution with the highest likelihood was the one finally chosen.

\subsection{Supervised Classification Models (Step 2)}

We tried to answer the following question: given a subset of SNPs, denoted by $Q \subseteq S$, to which extent are they associated to the gene whose expression level is modeled by the random varlable $G$, but using the varlable $H$ ?. More preclsely, we used supervised classification models [16] for this purpose. A supervised classification model learns a classification function from a pool of data samples, which is called the training data set or the training population. For this specific case, the classification function, $\mathrm{f}: \mathrm{Val}(\mathrm{Q}) \rightarrow$ $\mathrm{Val}(\mathrm{H})$, maps any posslble joint assignment, denoted by $\mathrm{q}$, of the varlables or SNPs in $Q \subseteq S$ to one value of the varlable $\mathrm{H}$.

In this work, we define the assoclation degree between the set of SNPs in $Q$ for a glven person and $H$ based on the quality with which the function $\mathrm{f}$ predicts the value of $\mathrm{H}$ when a joint assignment $q$ is known. In other words, we want to measure how well $\mathrm{f}$ predicts the discretized expression level (1.e. high or low) of the target gene knowing only the genotype of the SNPs in Q for that person.

A possible measure would be the so-called classification accuracy, which has to be estimated from a test data set or a test population, which is different of the training data set:

$$
\operatorname{Accuracy}(Q, M)=\frac{1}{T} \sum_{j=1}^{T} I\left[f\left(q_{j}\right)=h_{j}\right],
$$

where $T$ is the count of samples $\ln$ the test data set; $q_{j}$ and $h_{j}$ denote the joint value of the SNPs in Q and the value of $\mathbf{H}$ for the $j$-th member of the test data set, respectlvely; and I is the indicator function, which is equal to 1 if $f\left(q_{j}\right)$ is equal to $h_{1}$ (1.e., the prediction is correct) and 0 otherwise. Let us note, that the classification accuracy depends of the particular classification model $M$ and the subset of SNPs $Q$ we are evaluating. In this work, we also computed the Area Under the ROC Curve (AUC) [17] as a complementary and robust measure of the performance of a classifler.

Because our assoclation measure depends of the particular classification model, several state-of-the-art and computationally affordable classifiers were evaluated to get a more robust estimate of the association degree between SNPs and gene expression:

Naive Bayes [16]: It is a simple probabilistic classifier which works under the assumption that all input varlables are conditionally independent given the output varlable. As usual, predictions with this model are made by choosing the most probable class value given the genotype of an Individual.

C4.5 [18]: It is a classification tree model, in which the data set is divided in structured hypercubesof those Individuals sharing values. This is the most competitive algorlthm of this family. It is called J48 in an open-source version Implemented in Weka [19]. In this model, predictions are made by choosing the most frequent class in the leaf of the inferred tree where the genotype of the Individual to be classified falls.

Support Vector Machines (SVM): In this model the input varlables are transformed in a higher-dimension space so that a classifter is learned from the set of transformed variables by using a kernel function. We chose the default implementation of support vector machines in Wekausing the LibSVM java library [20]. 
One advantage of the first two approaches is that they build white-box models, i.e., models are directly readable and interpretable by human experts.

\subsection{Regression Models}

While a classification model predicts discrete or categorical values, a regression model makes continuous predictions. Similarly, it learns a regression function from a training data set. In this case, this regression function is defined as follows: g: $\operatorname{Val}(Q) \rightarrow \operatorname{Val}(G)$, where $\operatorname{Val}(G)$ corresponds to the real interval where the expression level of our target gene lies. We used this model to test the performance of the previously described "Continuous Model" (see Fig. 2), where no hidden variable it is assumed, for measuring SNP-expression level associations. Along the quality of the classification models, we used the quality of the continuous predictions of the $g$ function as a degree of association between the SNPs in Q and the gene G. A possible measure to evaluate the quality of a regression model is the root mean square error (RMSE) and the Pearson's correlation between the predicted and the real values [16], which has to be computed over a different test data set.

As it happened with the classification accuracy, this association measure depends on the particular regression model used. For this reason, we also considered a broad set of different regression models in our analysis:

SVM-reg [21]: It is based on support vector models and kernel methods, as its supervised classification counterpart.

Gaussian processes [22]: It is a Bayesian approach that employs Gaussian process priors over regression functions to improve their generalization capacity.

Lasso [23]: It applies regularization -a process of introducing additional information in order to solve an illposed problem or prevent overfitting- in the form of a penalty term for complexity, which performs variable selection by driving a number of regression coefficients to zero.

k-nearest neighbor [24]: It is an extension of the k-nn classifier which computes the output of a case by averaging the values of its $k$-nearest neighbors. In the simplest approach, where $k=1$, the assigned value is exactly the same as the one from the closest case. It is therefore advisable to use a $\mathrm{k}$ value bigger than one. Rightly so, it can be also useful to weight the contributions of the neighbors based on their distances to the case under evaluation. In our experiments, we used two values for $k$, namely $k=3$ and $k=$ 5 , and three different configurations to compute the output.

Let $\hat{y}$ be the regression estimator for a case $x$, and let $y_{1}$ be the value of the independent variable in the $i$-th case. The three approaches for the kNN-reg can be defined as follows:

1. Average value of the $\mathrm{k}$ nearest neighbors (kNN-regavg): $y=\frac{1}{k} \sum_{i=1}^{k} y_{i}$

2. Weighted estimation of the $\mathrm{k}$ nearest neighbors (kNN-reg-wgt): $y=\frac{\sum_{l=1}^{k} \frac{1}{D\left(x_{x} x_{i}\right.} y_{i}}{\left.\sum_{l=1}^{k} \frac{1}{D\left(x_{x}\right)}\right)}$ where $D\left(x, x_{l}\right)$ is the distance value between the case under evaluation, $x_{\text {, }}$ and $\mathbf{x}_{\mathbf{l}}$, one of its $k$ nearest neighbors.

3. Kernel density estimation ( $\mathrm{kNN}-\mathrm{reg}-\mathrm{krn}$ ): the output corresponds to the value for which a kernel density smoothing reaches its maximum density. The density function is estimated based on a normal kernel function, which takes the values of the $k$ nearest neighbors as input data.

As for measuring the distance between two genotypes, we made use of the Hamming distance. "The Hamming distance between two strings of equal length is the number of positions at which the corresponding characters are different" [25]. In genetic terms, it can be seen as the number of variations that transformed one genotype into the other.

\subsection{Comparing Classification and Regression Models}

As already commented, one of the main aims of this work was to evaluate how models which employ discretized expression values perform with respect to models which directly treat the expression as a real value. It is not straightforward to compare both approaches because they have different properties and, as commented in the above sections, the error measures usually employed to evaluate their performance are different. A possible approach to overcome this problem is to use the so-called relative absolute error (RAE). This metric evaluates a model by comparing the absolute error of the model itself with respect to the absolute error of a blinded model (i.e. the same model but not using any predictive variable: $Q=\emptyset$ ). This error metric is computed as follows:

$$
\begin{aligned}
& \text { RAE } \\
& =100 \cdot \frac{\sum_{j=1}^{T} \sum_{l=0}^{K}|\operatorname{Prediction}(j, l)-\operatorname{ActualValue}(j, l)|}{\sum_{j=1}^{T} \sum_{l=0}^{K}|\operatorname{PrlorPrediction}(l)-\operatorname{ActualValue}(j, l)|}
\end{aligned}
$$

For the regression models, $\mathrm{K}$ is equal to 1 ; Prediction( $(j, 1)$ corresponds to the real value prediction for the $j$-th test sample; ActualValue $(j, l)$ corresponds to the actual value associated to the same instance; and PriorPrediction(l) is the average value of the expression level in the training data set; this prediction does not depend of the particular individual because it does not use any knowledge about the SNPs (i.e. $\mathrm{Q}=\emptyset)$.

For the classification models, $\mathrm{K}$ is equal to the number of values of the discrete expression level. Thus, ActualValue(j, 1) $=1$ if the discretized expression value of the $j$-th instance of the test data set is equal to 1 , and 0 otherwise. Prediction( $j_{\text {, }}$ 1) with $1 \in\{1, \ldots, K\}$ is the prediction vector. For the Naive Bayes model it corresponds to the probabilities that this model assigns to each value of the discretized expression, because this is a probabilistic classifier which makes soft predictions. On the contrary, C4.5 and SVM models make hard predictions and, then, Prediction $(j, 1)=1$ for the predicted class and 0 otherwise. In this case, PrlorPrediction(1) is equal to the proportion of samples in the training population whose discretized expression value is equal to 1 . The above measure ranges from 0 to infinity. A zero value indicates a perfect prediction. Larger values indicate worse prediction capacity. A value over $100 \%$ 
Indicates strong overfltting because the model is performing worst than its blinded counterpart.

\subsection{Block Processing}

We were also interested to observe whether prediction accuracy changed when using models with a reduced number of SNPs, grouped by blocks of low recombination (1.e. SNPs with high linkage disequillbrlum (LD) among them). We grouped SNPs by using a common approach based on palrwise computations of confidence intervals of LD [14]. Palrwise LD is measured by the normalized statistic of allelic association $D^{\prime}$. The algorithm chooses the largest set of consecutive SNPs that reaches the requirements to be defined as a low recombination block, defined in terms of a minimum number of pairs of SNPs being in strong LD (onesided upper 95 European ancestrles than from Africa [21], we made blocks of chromosome 6 by using CEU, the data set of individuals with European ancestries (see Section 2.8) and used those blocks to group SNPs in YRI, the data set of Individuals with African ancestrles. Fig. (S2) shows the average number of SNPs by block in the data set used. Given a block, a classifler with only those SNPs in that block as input varlables was learned. As a result, SNPs in chromosome 6 were grouped in 345 non-overlapping blocks of low recombination [26], which were learned from the CEU data set. DRB5 is a gene coded between 11 physical positions 32593098 and 32606042 in assembly
NCBI36/hg18 or between 32485120 and 32498064 in assembly GRCh37/hg19. 6 SNPs has been genotyped in HapMap 3 within the gene DNA reglon. These SNPs belong to block 223. Tables 1 and 2 shows the SNPs within the block. Those in bold correspond to SNPs within the gene.

\subsection{An Overview of the Procedures}

Fig. (3) shows a flowchart with all the steps followed to conduct this study.

\subsection{Data Sets Used}

Expression data of gene DRB5 came from the mRNA of lymphoblastold cell lines of 228 individuals from different populations. Detalls about the procedure followed to obtain expression data, including raw expression data normallzation, population stratification correction andcorrection for known and unknown factors are described by Stranger et al. [3].

Several of these Indlviduals were genotyped by the International HapMap project [27]. We used the third phase [28] of HapMap project to obtain genotypes in order to avold large amounts of missing data, as in the other phases not all the individuals were genotyped. From all the CEU and YRI parental individuals in HapMap third phase, we only chose those CEU Individuals (107) and those YRI Individuals
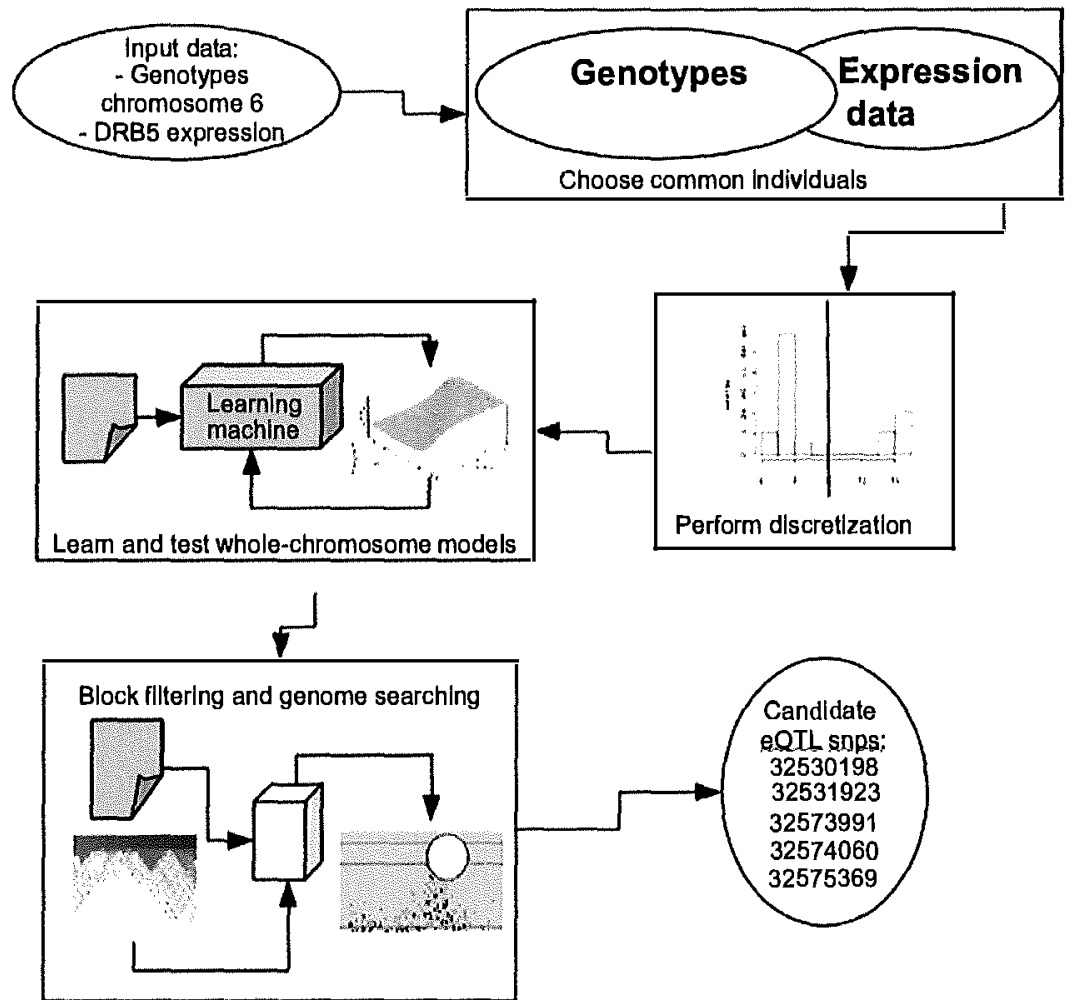

Fig. (3). Flowchart showing the steps followed in this study in order to obtaln a minlmal set of candidate eQTLs for expression of gene DRB5. 
Table 1. List of SNPs within block number 223. In bold those within the gene DNA region.

\begin{tabular}{|c|c|c|}
\hline rs Number & NCBI36/hg18 & GRCh37/hg19 \\
\hline $\begin{array}{c}\text { rs6901541 } \\
\text { rs17209754 } \\
\text { rs9269101 } \\
\text { rs9269110 } \\
\text { rs9378264 } \\
\text { rs1 } 12194148 \\
\text { rs9405112 } \\
\text { rs9378212 } \\
\text { rs } 9269182 \\
\text { rs } 4999342 \\
\text { rs } 4410767 \\
\text { rs35465556 } \\
\text { rs9378266 } \\
\text { rs9378213 } \\
\text { rs9269186 } \\
\text { rs9269187 } \\
\text { rs9269190 } \\
\text { rs9391786 } \\
\text { rs7748270 } \\
\text { rs7748472 } \\
\text { rs7749057 } \\
\text { rs7749242 } \\
\text { rs7749092 } \\
\text { rs6911871 } \\
\text { rs } 1964995 \\
\text { rs } 11752428 \\
\text { rs9269202 } \\
\text { rs } 11757500 \\
\text { rs9269204 } \\
\text { rs7754119 } \\
\text { rs9269211 } \\
\text { rs5020946 } \\
\text { rs } 12191360 \\
\text { rs } 1557551 \\
\text { rs6916742 } \\
\text { rs7754731 } \\
\text { rs288770027 }\end{array}$ & $\begin{array}{l}32550239 \\
32550681 \\
32550689 \\
32551247 \\
32551429 \\
32552176 \\
32553578 \\
32553669 \\
32555835 \\
32556076 \\
32556107 \\
32556112 \\
32556167 \\
32556376 \\
32556394 \\
32556418 \\
32556478 \\
32556539 \\
32556577 \\
32556741 \\
32556882 \\
32557008 \\
32557028 \\
32557256 \\
32557389 \\
32557448 \\
32557501 \\
32557633 \\
32557775 \\
32557836 \\
32558036 \\
32558067 \\
32559339 \\
32560168 \\
32561169 \\
32561832 \\
32574137\end{array}$ & $\begin{array}{l}32442261 \\
32442703 \\
32442711 \\
32443269 \\
32443451 \\
32444198 \\
32445600 \\
32445691 \\
32447857 \\
32448098 \\
32448129 \\
32448134 \\
32448189 \\
32448398 \\
32448416 \\
32448440 \\
32448500 \\
32448561 \\
32448599 \\
32448763 \\
32448904 \\
32449030 \\
32449050 \\
32449278 \\
32449411 \\
32449470 \\
32449523 \\
32449655 \\
32449797 \\
32449858 \\
32450058 \\
32450089 \\
32451361 \\
32452190 \\
32453191 \\
32453854 \\
32466159\end{array}$ \\
\hline $\begin{array}{c}\text { rs } 35847514 \\
\text { rs } 34507021 \\
\text { rs } 1137498 \\
\text { rs } 35085841 \\
\text { rs } 35056680 \\
\text { rs } 35739325 \\
\text { rs } 34328528 \\
\text { rs } 17203992 \\
\text { rs } 16870207 \\
\text { rs2157337 } \\
\text { rs } 342496660 \\
\text { rs } 28772724 \\
\text { rs } 28760027 \\
\text { rs } 2157339 \\
\text { rs } 284953556 \\
\text { rs } 35571839 \\
\text { rs } 34369284 \\
\text { rs } 28490179 \\
\text { rs } 11759557 \\
\text { rs } 11757159 \\
\text { rs } 34781832 \\
\text { rs } 1064611 \\
\text { rs } 34569694 \\
\text { rs } 28656080 \\
\text { rs } 28530648 \\
\text { rs } 35464393 \\
\text { rs } 12661707 \\
\text { rs } 35366052\end{array}$ & $\begin{array}{l}32587470 \\
32587485 \\
32593190 \\
32597079 \\
32601256 \\
32601768 \\
32601789 \\
32604788 \\
32606390 \\
32609122 \\
32609486 \\
32617335 \\
32617963 \\
32619650 \\
32620142 \\
32620591 \\
32622122 \\
32626983 \\
32628011 \\
32628250 \\
32628606 \\
32630503 \\
32632659 \\
32633666 \\
32635057 \\
32638176 \\
32639223 \\
32639901\end{array}$ & $\begin{array}{l}32479492 \\
32479507 \\
32485212 \\
32489101 \\
32493278 \\
32493790 \\
32493811 \\
32496810 \\
32498412 \\
32501144 \\
32501508 \\
32509357 \\
32509985 \\
32511672 \\
32512164 \\
32512613 \\
32514144 \\
32519005 \\
32520033 \\
32520272 \\
32520628 \\
32522525 \\
32524681 \\
32525688 \\
32527079 \\
32530198 \\
32531245 \\
32531923\end{array}$ \\
\hline
\end{tabular}

(107), for which we had the expression of gene DRB5. We chose the SNPs passing quality control which overlapped genes DRB5 and DRB1 or within a window of 1 million basis before the first gene position (28922491 in assembly GRCh37/hg19) and after the last gene position (33961785 in assembly GRCh37/hg19) in chromosome 6 (where genes DRB5 and DRB1 belong to). The total number of SNPs was 6593. Missing information was inferred by using familial information and the IMPUTE2 algorithm [29] and these data were downloaded from the HapMap project website (http: //www.hapmap.org). The final two data sets were called CEU-array and YRI-array. For a second analysis pursuing to replicate results using expression data obtained by nextgeneration RNA sequencing (NGS) technology, we built other two data sets (CAU-RNASeq and YRI-RNASeq). Expression data for gene DRB5 was obtained from the Geuvadis project [30], a second experiment using NGS technology in which the RNA of 465 lymphoblastold cell lines from the 1000 Genomes project [31] was sequenced. To accurately quantify the expression level of genes from RNA-seq reads we first applied a cleaning procedure on raw data followed by a common protocol TopHat-Cufflink [32] to obtain gene expression levels. Thus, from the cleaned data (files if FASTQ format) at the Geuvadis project we used TopHat software, "a read alignment program that allows alignments between a read and the genome to contain large gaps" [32], to perform read alignment using the human genome as reference. "To assemble individual transcripts from the RNA-seq reads that were aligned to the genome and obtain the expression level of genes" [32], we used Cufflinks. Out of these 465 cell lines, 259 belonged to Caucasian individuals (only 82 of them were included in the data used in our first analysis, the others correspond to British individuals) and 79 were the only African individuals in the study, all of them Yoruban included in the data used in our first analysis. To avoid reducing sample size of the CEU data from 259 to 79 , we decided to make two data sets with the 259 Caucasian and the 79 African individuals by using genotypes from 1000 Genomes as the other individuals were not genotyped by the HapMap project. SNP selection was made following the same criterion as with HapMap data. The total number of selected SNPs, 97, 484, was much higher due to the higher genotyping density used by the 1000 Genomes project. Finally, and in order to understand the lower performance in results when usingRNASeq data, we also created two data sets, CEU-commonIndividuals, YRIcommonIndividuals with respectively only the $82 \mathrm{CEU}$ and 81 YRI whose DRB5-expression was obtained by the two different gene expression technologies.

\section{RESULTS}

\subsection{Microarray Expression Data ${ }^{1}$}

\subsubsection{Discretization Step}

The result of the discretization step on the microarray gene expression via the EM algorithm was clear for the CEU

'A reduced version of these experiments with microarray expression data were presented in the conference paper's version of this work. We present again some of the results for the sake of completeness and readability of the paper. 
and YRI populations. The EM algorithm defined two groups which could be easily identified by looking at the histograms shown in Fig. (1). Before comparing multivariate models, and in order to understand how discretization behaved when using the common single-SNP association, we first compared, for each one of the 6593 SNPs considered in CEU-array and YRI-array data sets, the Spearman correlation coefficients obtained when using the continuous expression of the DRB5 gene and when using the discretized variable. For this last case, the binary variable was assumed to take two real values: 0 for low-expression; and 1 for highexpression. In Fig. (4), we plot the Spearman correlation coefficients of this comparison for the two populations.

As can be seen in this figure, the " 2 Bins Discretization" series and the "Continuous Value" series are quite similar. Actually, if we compute the Spearman correlation coefficient

(a) CEU Microarray data

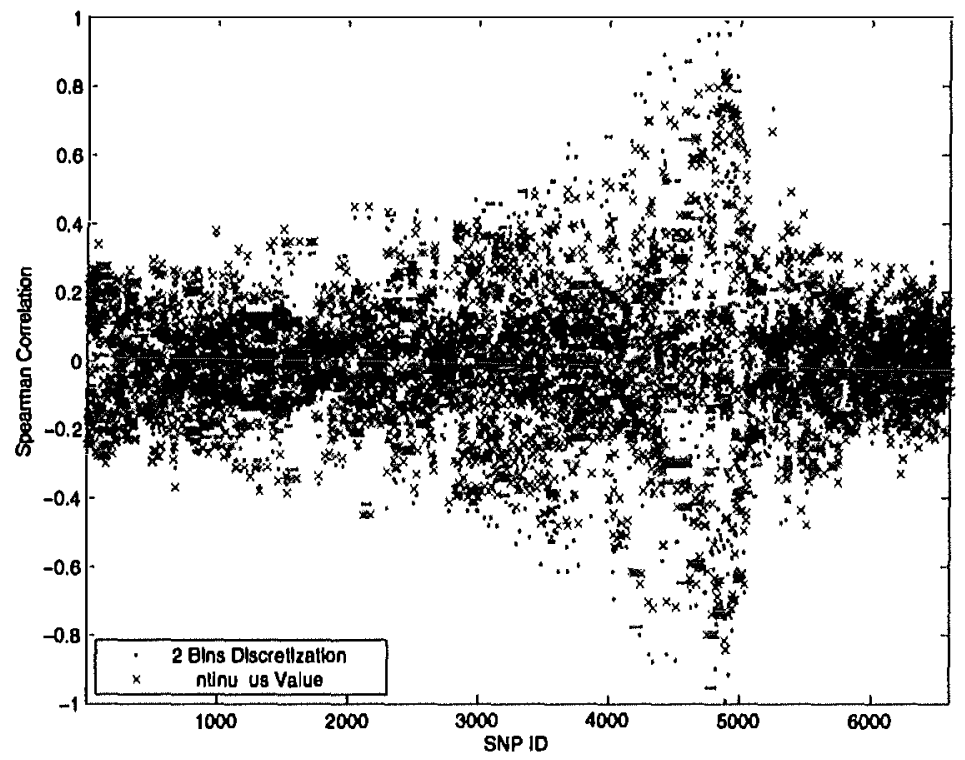

(b) YRI Mlcroarray data

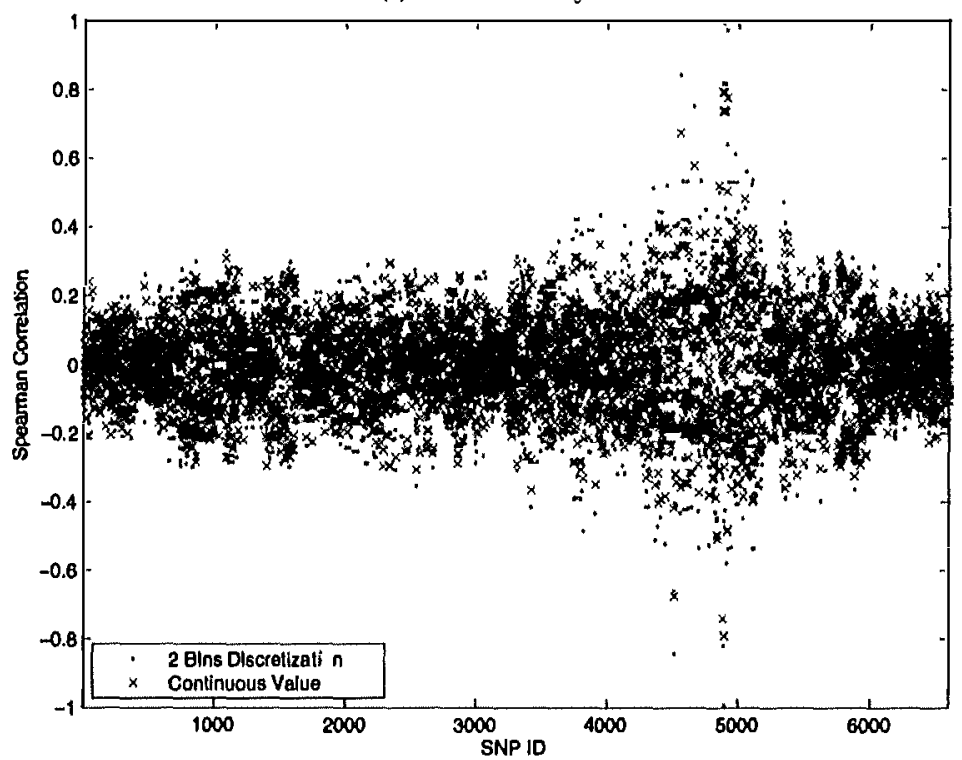

Fig. (4). Spearman correlation for each 6593 analyzed SNPs and the continuous expression level (blue points); and for the same SNPs and the binarized expression (red points). The Spearman correlation value between the continuous and discrete serles of CEU-array was equal to 0.8938 ; for YRI-array was equal to 0.8798 . 
Table 3. The 10 SNPs with the highest Spearman correlation coefficients for the two populations computed when the expression of the gene is continuous and discretized. Those four SNPs among the 10 top SNPs in both populations are highlighted.

\begin{tabular}{|c|c|c|c|c|c|c|c|}
\hline \multicolumn{4}{|c|}{ YRI-Array } & \multicolumn{4}{|c|}{ CEU-Array } \\
\hline \multicolumn{2}{|c|}{ Discrotized } & \multicolumn{2}{|c|}{ Continuous } & \multicolumn{2}{|c|}{ Diseretized } & \multicolumn{2}{|c|}{ Cont inuous } \\
\hline Pou & Corr. & Pou & Corr. & Pou & Corr. & Pou & Corr. \\
\hline 32638176 & 0.9981 & 32638176 & 0.7944 & 32639901 & 1 & 32681969 & 0.8413 \\
\hline 32639901 & 0.9981 & 32639901 & 0.7944 & 32638176 & 0.995 & 32682038 & -0.8413 \\
\hline 32682038 & -0.9946 & 32681969 & 0.792 & 32681969 & 0.9905 & 32683347 & -0.8413 \\
\hline 32683347 & -0.9946 & 32682038 & -0.7904 & 32682038 & -0.9905 & 32638176 & 0.8373 \\
\hline 32681969 & 0.9931 & 32683347 & -0.7904 & 32683347 & -0.9905 & 32639901 & 0.8337 \\
\hline 32694832 & 0.9727 & 32694832 & 0.7759 & 32500884 & 0.9517 & 32648019 & 0.82 \\
\hline 32396216 & -0.8431 & 32666924 & 0.7411 & 32500959 & -0.9517 & 32666924 & 0.8151 \\
\hline 32417132 & 0.8431 & 32678817 & -0.7411 & 32512355 & -0.9517 & 32678817 & -0.8151 \\
\hline 32666924 & 0.8193 & 32648019 & 0.7372 & 32518965 & -0.9517 & 32684456 & 0.8151 \\
\hline 32678817 & -0.8193 & 32684456 & 0.7372 & 32521029 & -0.9517 & 32685867 & 0.8151 \\
\hline
\end{tabular}

for these two pair of serles (1.e. treating the Spearman coefficients between SNPs and gene expression as two independent serles of real values) we find that the ' 2 Bins Discretization" serles and the "Continuous Value" serles were highly correlated. For CEU population the correlation was equal to 0.8938 while for YRI population was equal to 0.8798. That means that those SNPs that are highly correlated with the contlnuous expression of the gene are also similarly correlated with the discretized expression of the gene. In Table 3 we detall the 10 SNPs with the highest Spearman correlation coefficients for the two populations computed when the expression of the gene is contlnuous and discretized. As it can be seen, with the discretized expression of the gene the correlation notably Increased in both populations.

As summary, we find a positive effect on the Spearman correlation coefficlents when discretizing the DRB5 microarray expression level.

\subsubsection{Whole Models}

We later evaluated classification and regression models using all SNPs at a time (1.e. $Q=S$ ) to study the extent to which the expression level using microarrays of DRB5 is controlled by the selected SNPs. To correctly evaluate the performance of classification and regression models, we employed the so-called 10-fold cross-validation methodology $(10-\mathrm{cv})$ [33] to bulld different training and test data sets: firstly, the members of one data set are randomly divided in 10 groups; then, ten different test data sets are created by selecting each time a different group; the other ten different training sets are bullt with the remaining 9 groups; finally, the models are trained and tested ten times with each train/test pair and the averaged performance measures of these ten validations are reported.

Tables 4 and 5 show respectively results for data sets CEU-array and YRI-array. The relatlve absolute error (see Section 2.5) is defined for both classification and regression models. The application of a palred t-test reveals that RAE for the algortthm with best results under the discretization approach (C4.5) is significantly lower than the best algorlthm among the common regression approach (Lasso): $\mathrm{p}$ values are $1.0 \mathrm{e}-6$ and $1.0 \mathrm{e}-4$ for CEU and YRI data sets respectlvely. Again, a non-parametrlc test such as Wilcoxon could have been also applied.

Table 4. Generalization capacity of different classification and regression models using all SNPs as input variables in CEU-array data set.

\begin{tabular}{|c|c|c|c|}
\hline Classification Modols & Accuracy & AUC & Rolat. Abs. Error \\
\hline \hline NB & 91,64 & 0.89 & 18.58 \\
C4.5 & $\mathbf{1 0 0 . 0}$ & $\mathbf{1 . 0 0}$ & $\mathbf{0 . 0 0}$ \\
SVM & 89.92 & 0.86 & 21.87 \\
\hline Rogrossion Modols & RMSE & Corrolation & Rolat. Abs. Error \\
\hline \hline SVM-Reg. & $\mathbf{1 . 5 6}$ & 0.87 & 37.27 \\
Gaussian Proc. & $\mathbf{1 . 5 6}$ & 0.87 & 37.28 \\
Lasso & $\mathbf{0 . 4 1}$ & $\mathbf{0 . 9 9}$ & $\mathbf{1 0 . 4 7}$ \\
3-NN-reg-avg & 2.74 & 0.52 & 60.78 \\
5-NN-reg-avg & 2.66 & 0.54 & 62.47 \\
3-NN-reg-wgt & 2.73 & 0.53 & 60.55 \\
5-NN-reg-wgt & 2.65 & 0.55 & 62.17 \\
3NN-reg-krn & 3.12 & 0.57 & 60.12 \\
5NN-reg-krn & 2.99 & 0.61 & 54.62 \\
\hline
\end{tabular}


Looking at these results, we can observe that $\mathrm{C} 4.5$ has an outstanding performance because it achleves perfect or almost perfect classification in CEU-array and YRI-array data sets. These are very good news because C4,5 is not a black-box machine learner. Its decision tree based nature allows to easily interpret the classification rules used for making predictions. So, this model could potentlally help blomedical researchers to understand SNPs regulation of DRB5 expression. On the contrary, classifiers based on SVM and regression models had much worst predictlve performance (1.e. higher relative absolute error). However, a much better performance was expected due to the wellknown fact that expression regulation of DRB5 is controlled by genetlc varlants in chromosome 6 tagged by some of the SNPs from the genotype array used.

We see again how the discretization step bullt more accurate prediction models than considering the gene expression as a continuous value and, hence, using regression techniques.

However, it has to be noted that Lasso regression clearly outperforms the other regression methods and it also outperforms SVM classifiers. A further discussion of this result is given in Section 4.

Finally, we want to comment that when Inspecting the tree model learnt with the $\mathrm{C} 4.5$ algorithm we can see that, etther for CEU-array or for YRI data sets, we obtaln a simple tree with one single attribute. This single attribute is different for each population but it belongs in both cases to the block 223. Further analyses at this respect are given in following section.

\subsubsection{Block-Based Approach}

In this new analysis we depart from the block partition described in Section 2.6. With this analysis, we tried to understand which blocks are more correlated with the microarray expression of DRB5. For this purpose, we evaluated the performance of the classification and regression models by using as input variables (1.e. Q) those SNPs contained in a single block. This is a blologically-
Inspired feature selection method. Would accuracy keep as higher as when all SNPs were used in classification/ regression models? Would it increase because some nolse or redundant varlables were eliminated?

Among the classification models, we plcked the C4.5 model, the one which performed best, as shown in the prevlous section. The Lasso regression model was chosen from the same reason among the regression models. We built a total of 345 data sets for each population by selecting the SNPs within each block and used the $10-\mathrm{cv}$ evaluation method to estimate the different performance measures.

In Fig, (5), we display the results of this analysis. In Table 6 we detall the 10 blocks with the lowest relative absolute error for the classification and the regression model in the two populations. It can be seen again that classification models performed in both populations better than regression models in the key SNPs blocks, which are highly associated to the expression of the DRB5.

Another question that arises in this analysis is whether the performance of a classification model using a set of SNPs is higher or not than the performance obtalned using a single SNP. That is to say, can we predict better the expression level of DRB5 by aggregating multiple SNPs? To try to answer this question, we compared AUC of the C4.5 classification model using all SNPs within the same block with respect to the AUC obtained using only one single SNP. We selected the SNP with the maximum AUC within the same block. In Fig. (6) we plot both measures for all the 345 blocks. In Table 7 we detall the 10 blocks with the highest AUC using the C 4.5 classifler as well as the AUC obtained with the best performing single SNP among all SNPs within this block. These results are deeply discussed in Section 4 .

\subsection{Replication with RNASeq Expression Data}

\subsubsection{Discretization Step}

The discretization of the two RNASeq-based populations, CAU-RNASeq and YRI-RNASeq, were much less stralghtforward than the array-based populations. As can be

Table 5. Generalization capacity of different classification and regression models using all SNPs as input variables in YRI-array data set.

\begin{tabular}{|c|c|c|c|}
\hline Classification Models & Accuracy & AUC & Relat. Abs. Error \\
\hline $\begin{array}{c}\text { NB } \\
\text { C4.5 } \\
\text { SVM }\end{array}$ & $\begin{array}{l}89,7 \\
99.0 \\
79,5\end{array}$ & $\begin{array}{l}0.89 \\
1.00 \\
0.66\end{array}$ & $\begin{array}{c}24.53 \\
1.66 \\
48.43\end{array}$ \\
\hline Regression Models & RMSE & Corrolation & Relat. Abs. Error \\
\hline $\begin{array}{c}\text { SVM-Reg. } \\
\text { Gaussian Proc. } \\
\text { Lasso } \\
\text { 3-NN-reg-avg } \\
\text { 5-NN-reg-avg } \\
\text { 3-NN-reg-wgt } \\
\text { 5-NN-reg-wgt } \\
\text { 3NN-reg-krn } \\
\text { 5NN-reg-km }\end{array}$ & $\begin{array}{l}1.99 \\
1.99 \\
0.74 \\
2.66 \\
2.62 \\
2.68 \\
2.62 \\
2.34 \\
2.68\end{array}$ & $\begin{array}{l}0.76 \\
0.76 \\
0.98 \\
0.54 \\
0.50 \\
0.53 \\
0.50 \\
0.63 \\
0.52\end{array}$ & $\begin{array}{l}55.06 \\
55.05 \\
17.29 \\
69.28 \\
72.26 \\
69.65 \\
72.33 \\
46.53 \\
51.37 \\
\end{array}$ \\
\hline
\end{tabular}


Table 6. The 10 blocks with the lowest relative absolute error (RAE) using a classification model (C4.5) learnt with all SNPs within this block. 7 blocks (in bold) out of the 10 are shared by the two populations.

\begin{tabular}{|c|c|c|c|c|c|c|c|}
\hline \multicolumn{4}{|c|}{ YRI-Array } & \multicolumn{4}{|c|}{ CEU-Array } \\
\hline \multicolumn{2}{|c|}{ Discretized } & \multicolumn{2}{|c|}{ Continuous } & \multicolumn{2}{|c|}{ Discrotized } & \multicolumn{2}{|c|}{ Continuous } \\
\hline Block Id & RAE & Block Id & RAE & Block Id & RAE & Block Id & RAE \\
\hline 224.00 & 0.00 & 223.00 & 12.85 & 221.00 & 0.00 & 223.00 & 8.34 \\
\hline 225.00 & 0.00 & 225.00 & 23.62 & 223.00 & 0.00 & 224.00 & 20.27 \\
\hline 223.00 & 1.56 & 224.00 & 24.84 & 224.00 & 0.00 & 225.00 & 20.37 \\
\hline 227.00 & 4.39 & 227.00 & 26.72 & 226.00 & 7.79 & 221.00 & 24.44 \\
\hline 226.00 & 9.08 & 226.00 & 31.38 & 215.00 & 7.86 & 227.00 & 24.72 \\
\hline 214.00 & 25.48 & 213.00 & 45.16 & 216.00 & 7.86 & 226.00 & 28.08 \\
\hline 203.00 & 28.49 & 203.00 & 45.62 & 219.00 & 7.86 & 216.00 & 28.11 \\
\hline 205.00 & 28.49 & 205.00 & 48.15 & 227.00 & 7.86 & 219.00 & 28.12 \\
\hline 221.00 & 30.22 & 198.00 & 54.17 & 225.00 & 8.50 & 215,00 & 28.42 \\
\hline 213.00 & 32.62 & 221.00 & 54,63 & 203.00 & 19.26 & 203.00 & 38.81 \\
\hline
\end{tabular}

(a) CEU Microarray data

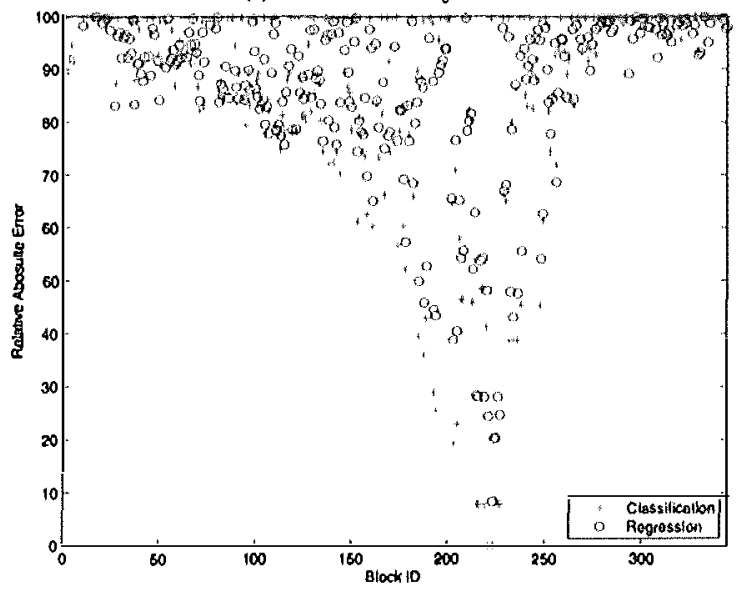

(b) YRI Microarray data

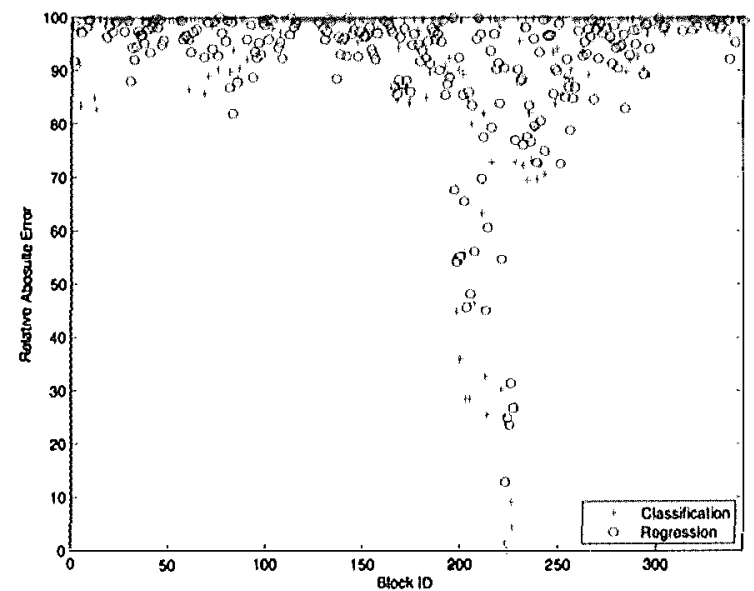

Fig. (5). Relative Absolute Error comparison between a classification model (C4.5, red plus sign) and a regression model (Lasso, blue clrcle). For the CEU-array population, the number of blocks where the classification model obtains a RAE < $10 \%$ is 9 and for RAE < $30 \%$ the number of blocks is 13 . For the regression model in the CEU-array population, these numbers are 1 and 9 respectively. For the YRl-array population, the number of blocks where the classiflcatlon model obtalns a RAE $<10 \%$ is 5 and for RAE $<30 \%$ the number of blocks is 8 . For the regression model in the YRI-array population, these numbers are 0 and 4 respectively. 
Table 7. The 10 blocks with the highest AUC using a classification model (C4.5). "Single SNP AUC" column displays the AUC obtained with the best performing single SNP among all SNPs within this block. 7 blocks (in bold) out of the 10 are shared by the two populations.

\begin{tabular}{|c|c|c|c|c|c|}
\hline \multicolumn{3}{|c|}{ YRI-Array } & \multicolumn{3}{c|}{ CEU-Array } \\
\hline \hline Block ID & Block AUC & Singlo SNP AUC & Block ID & Block AUC & Singlo SNP AUC \\
\hline $\mathbf{2 2 3}$ & 1.000 & 1.000 & $\mathbf{2 2 1}$ & 1.000 & 0.866 \\
$\mathbf{2 2 4}$ & 1.000 & 1.000 & $\mathbf{2 2 3}$ & 1.000 & 1.000 \\
$\mathbf{2 2 5}$ & 1.000 & 0.668 & $\mathbf{2 2 4}$ & 1.000 & 1.000 \\
$\mathbf{2 2 6}$ & 0.973 & $\mathbf{2 2 5}$ & 0.983 & 0.760 \\
$\mathbf{2 2 7}$ & 0.970 & 0.970 & $\mathbf{2 2 6}$ & 0.973 & 0.697 \\
$\mathbf{2 2 1}$ & 0.899 & 0.689 & 215 & 0.954 & 0.954 \\
213 & 0.892 & 0.741 & 216 & 0.954 & 0.954 \\
214 & 0.858 & 0.678 & 219 & 0.954 & 0.954 \\
$\mathbf{2 0 3}$ & 0.855 & 0.839 & $\mathbf{2 2 7}$ & 0.954 & 0.954 \\
205 & 0.855 & 0.839 & $\mathbf{2 0 3}$ & 0.899 & 0.897 \\
\hline
\end{tabular}

seen in the count-histograms shown in Fig. (7), there were not so clearly differentiated groups as in the case of arraybased data sets. This has opened an issue still under research that will be discussed in Section 4 .

The first declsion we had to make to proceed with the application of the EM algorithm for discretizing these data sets was to choose the number of bins (1.e. the $K$ value). The problem here is that EM is not able to directly indicate which is the optimal number of blns. Although some methods has been proposed to help EM to decide the number of bins [34], there is not clear solution to this problem and the best approach usually is a mixture of using some expert knowledge, If avallable, and trlal-error tests. In our case, we evaluated two and three bins discretization configurations.

In a first analysis, we compared the Spearman correlation coefficients obtained between each one of the 97, 484 SNPs considered in this data set and the continuous expression of DRB5 gene, and between the same SNPs and the discretized expression in two and three bins obtained with the EM al gorlthm. For these last two cases, the 2 bins varlable was assumed to take two values: 0 for low-expression; and 1 for high-expression; and the 3 bins varlable was assumed to take three values: 0 for the lowest expresslon group; 1 for the middle expression group; and 2 for the highest expression group. In Fig. (8), we plot the Spearman correlation coefficients of this comparison for the two populations.

As it can be seen in this flgure, the "2 Bins Discretization" serles, the " 3 Bins Discretization" serles and the "Continuous Value" serles are not very different among them. When we computed the Spearman correlation coefficient between "Continuous Value" serles and the "3 Bins Discretization" serles (i.e. treating the Spearman correlation coefficients between SNPs and gene expression as real values) we found that they were highly correlated: for CEU population the correlation was equal to 0.9636 while for YRI population was equal to 0.9315 . When we performed this analysis with the "2 Bins Discretization" the Spearman correlation coefficlents were 0.9319 and 0.9218 for CAU-RNASeq and YRI-RNASeq, respectively.
In Tables 8 and 9, we detall the 10 SNPs with the highest Spearman correlation coefficlents for the two populations computed when the expression of the gene is continuous and discretized in 2 and 3 bins. As can be seen, the discretization with 3 bins generates higher Spearman correlation between SNPs and gene expression than 2 bins discretization. We can also see like the correlation with the discretized expression does not strongly increase as happens with the microarray data.

In light of the above results we decided to continue with 3 bins discretization. In Fig. (9) we show the Gaussian mixtures inferred by the EM algorlthm using 3 Gaussian components for the two data sets.

\subsubsection{Whole Models}

As we did with microarray data, we evaluated again the classification and regression models using all SNPs at a time (1.e. $\mathrm{Q}=\mathrm{S}$ ) to study the extent to which the RNASeq expression of DRB5 is controlled by the new selected SNPs.

Tables $\mathbf{1 0}$ and $\mathbf{1 1}$ show respectively results for data sets CAU-RNASeq and YRI-RNASeq, following the same evaluation methodology used in Section 3.1.2. The application of a paired t-test at 0.05 level reveals that SVM, the algortthm with the best results among those under the discretization approach, has RAE significantly lower than the one reached by the algorthm under the common regression approach (Lasso): $p$ values are 0.0396 and 0.0068 for CEU and YRI respectlvely. A non-parametrlc test such as Wilcoxon could have been also applied.

Looking at these tables, we can see that both the classification and the regression models performs poorly than in the case of microarray data. In Section 4 we discuss about possible reasons of RNASeq underperforming microarrays, when it is supposed to be a more accurate technology [34]. However, the classification models still perform better than the regression models when comparing their relative absolute errors. Another unexpected result is that SVM outperform C4.5 in YRI, even when it does not perform any varlable selection procedure. Again it may be 
(a) CEU Microarray data

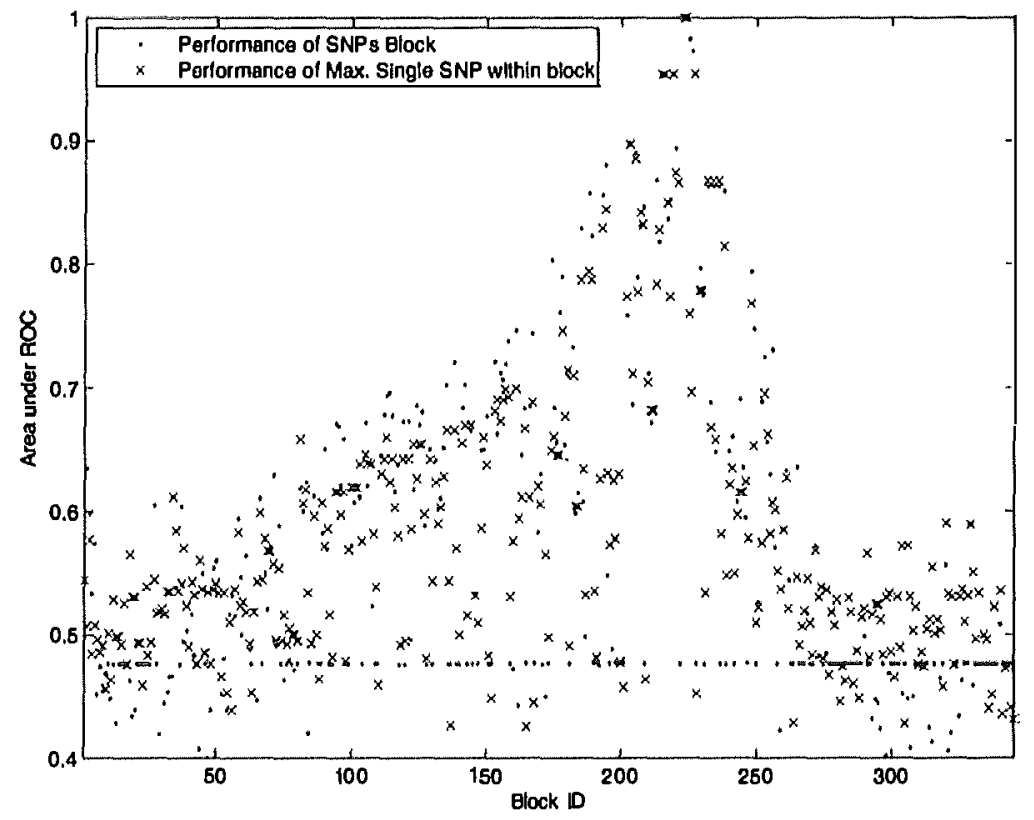

(b) YRI Microarray data22

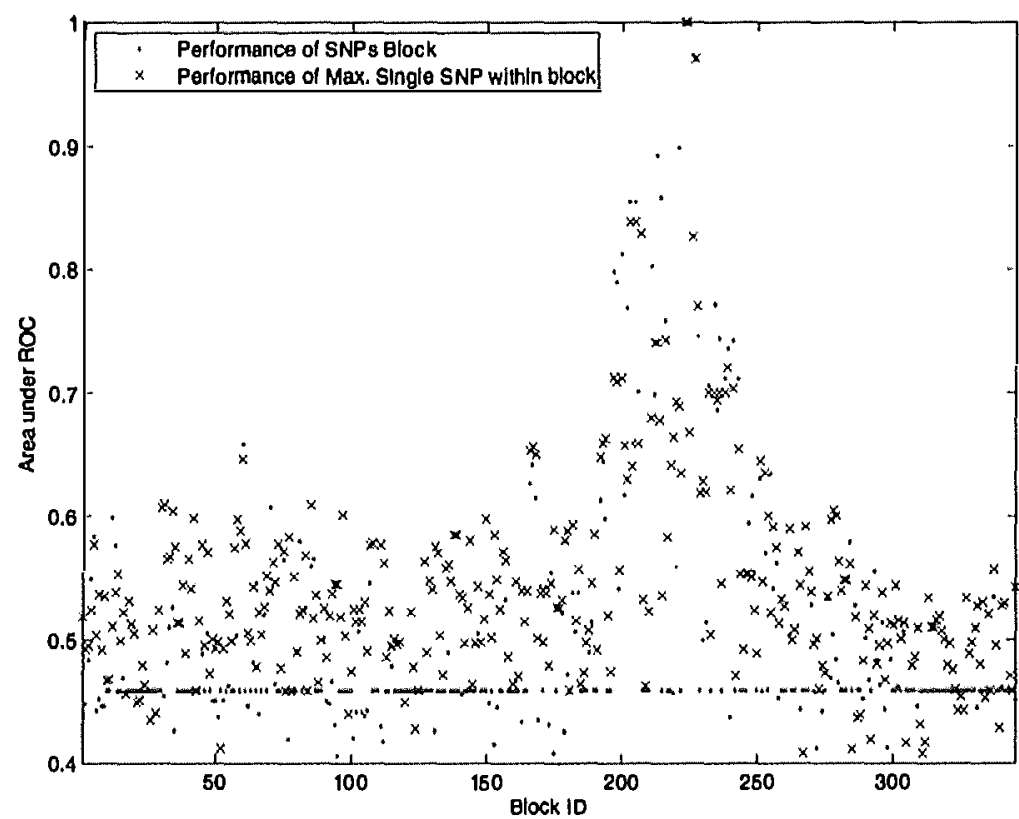

Fig. (6). AUC comparison between a classification model (C4,5) using all SNPs in a block (red flled clrcle); and the maximum performance obtalned using a single SNP within the same block (blue cross).

due to a problem in the way expression data was obtained by RNASeq technology and will be discussed in Section 4. Like in the case of microarray data, we trled to inspect the tree models induced by C4.5 using the CAU-RNASeq and the YRI-RNASeq populations. However in this case trees are not as easily interpretable as before because they involved tens of different SNPs and, moreover, they tend to vary if these trees are induced with slightly different training data sets, as happens when using cross validation. 
DABS-RotBoqCEU

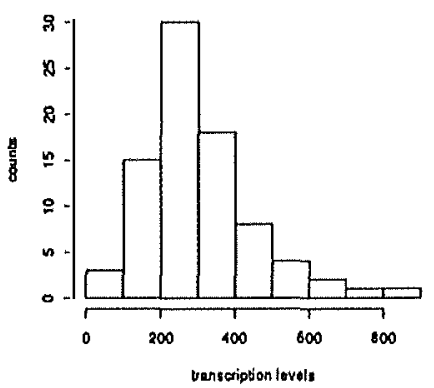

DRB5. e e -Yค!

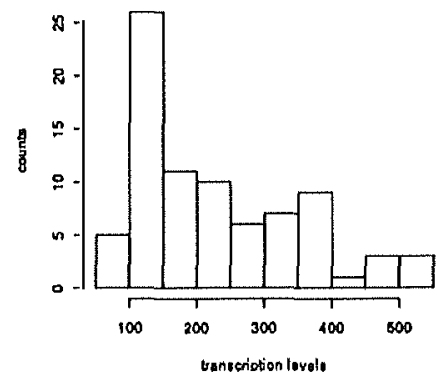

Fig. (7). Count-histograms of transcription levels ( $x$-axis) for DRB5 in CAU-RNASeq data set (a) and YRI-RNASeq data set (b).

(a) CAU RNASeq data

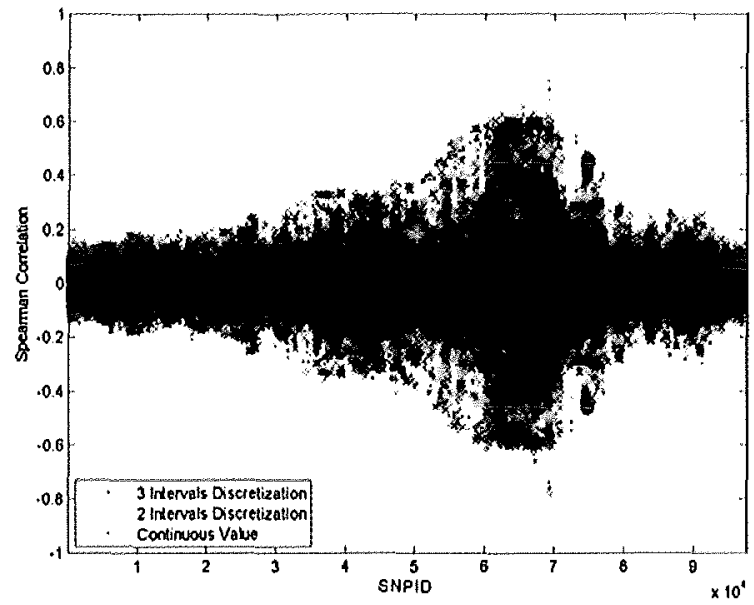

(b) YRI RNASeq data

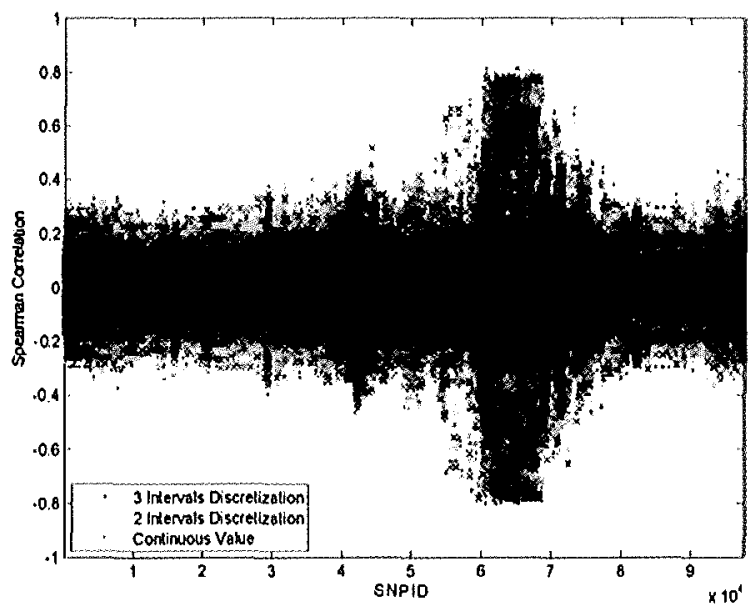

Fig. (8). Spearman correlation between 97, 484 analyzed SNPs and the continuous expression level (blue points); between the SNPs and the 3 bins discretization expression (red polnts); and between the SNPs and the 2 blns discretization expression (cyan polnts). For the CAU population, the Spearman corr elation value between the contlnuous and the 2 bins series was 0.9319 ; and between the continuous and the 3 bins serles was 0.9636 . For this same CAU population, the averaged mean square error between the continuous and the 2 bins serles was 0.0013 ; and between the continuous and the 3 bins serles was 0.000055 . For the YRI population, the Spearman correlation value between the continuous and the 2 binsseries was 0.9218 and between the contInuous and the 3 bIns serles was 0.9315 . For this same YRI population, the averaged mean square error between the continuous and the 2 bins serles was 0.0020; and between the continuous and the 3 bins serles was 0.0018 . 
Table 8. The 10 SNPs with the highest Spearman correlation coefficients for the YRI-RNASeq population computed when the expression of the gene is continuous and discretized in two and three bins.

\begin{tabular}{|c|c|c|c|c|c|}
\hline \multicolumn{6}{|c|}{ YRI-RNASeq } \\
\hline \multicolumn{2}{|c|}{ Discretlzed 3 Blns } & \multicolumn{2}{|c|}{ Discretlzed 2 Bins } & \multicolumn{2}{|c|}{ Contlnuous } \\
\hline Pou & Corr. & Pou & Corr. & Pou & Corr. \\
\hline 32458003 & 0.8132 & 32486115 & -0.7741 & 32533567 & 0.8126 \\
\hline 32533567 & 0.8124 & 32458228 & 0.7713 & 32561743 & 0.7973 \\
\hline 32458007 & -0.8007 & 32502393 & -0.7682 & 32533813 & -0.793 \\
\hline 32486115 & -0.7993 & 32491826 & -0.7663 & 32537290 & -0.793 \\
\hline 32492845 & -0.7971 & 32502507 & -0.7657 & 32486115 & -0.7927 \\
\hline 32458228 & 0.7952 & 32502513 & -0.7657 & 32481244 & -0.7917 \\
\hline 32561743 & 0.7894 & 32502522 & 0.7633 & 32486632 & 0.7908 \\
\hline 32486639 & 0.7821 & 32492845 & -0.7627 & 32533755 & -0.7878 \\
\hline 32533813 & -0.7821 & 32533567 & 0.7587 & 32553705 & -0.7864 \\
\hline 32537290 & -0.7815 & 32481244 & -0.7528 & 32553531 & -0.7864 \\
\hline
\end{tabular}

Table 9. The 10 SNPs with the highest Spearman correlation coefficients for the CAU-RNASeq population computed when the expression of the gene is continuous and diseretized in two and three bins.

\begin{tabular}{|c|c|c|c|c|c|}
\hline \multicolumn{2}{|c|}{ Discretlzed 3 Blns } & \multicolumn{2}{|c|}{ Discretized 2 Blns } & \multicolumn{2}{|c|}{ Continuous } \\
\hline 32602872 & -0.757 & 32602872 & -0.7171 & 32602872 & -0.7831 \\
\hline 32602396 & -0.7371 & 32602396 & -0.7042 & 32602396 & -0.7637 \\
\hline 32540158 & 0.6251 & 32486683 & -0.5634 & 32540158 & 0.6511 \\
\hline 32546592 & -0.6029 & 32489908 & 0.5615 & 32561424 & -0.637 \\
\hline 32561743 & 0.6005 & 32488193 & -0.548 & 32561743 & 0.6307 \\
\hline 32545106 & -0.6005 & 32479606 & -0.547 & 32489908 & 0.6267 \\
\hline 32599071 & 0.5964 & 32501522 & 0.5411 & 32599071 & 0.6232 \\
\hline 32534976 & 0.5949 & 32454968 & -0.5392 & 32454968 & -0.6231 \\
\hline
\end{tabular}

(a) CAU RNASeq data

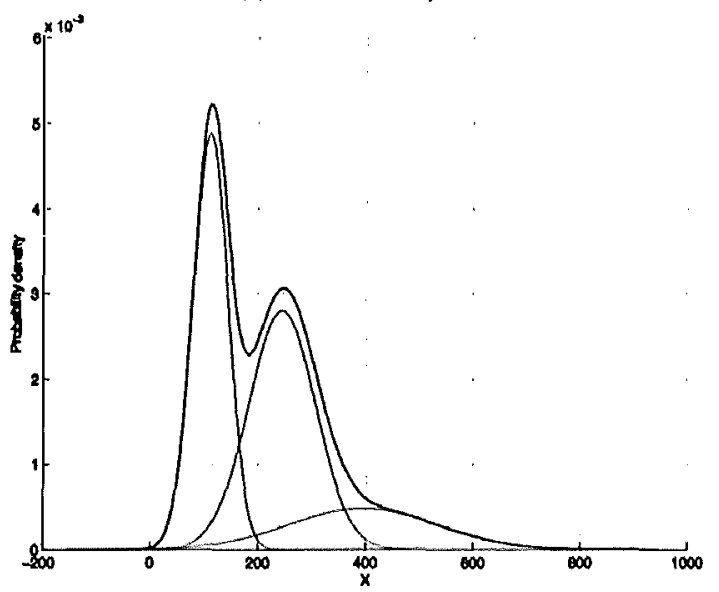

(b) YRI RNASeq data

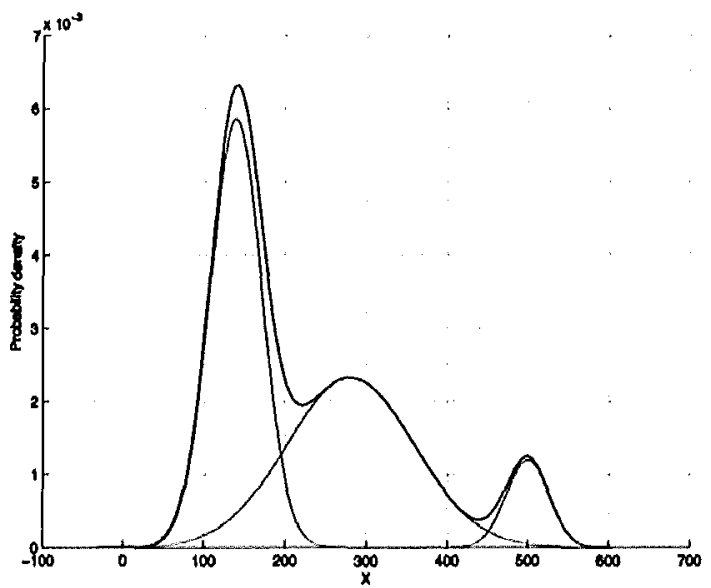

Fig. (9). Gaussian mixturcs estimated by the EM algorithm. EM was run 100 times with different random starting points, the solution with best likelihood was chosen. For the CAU-RNASeq population, the mixture model defined two cut-off points: 166.00 and 361.97 ; and these cut-off points created 3 groups with 118,110 and 31 individuals inside each group. For the YR1 population, the mixture model defined two cut-off points: 194.39 and 451.11 ; these cut-off points created 3 groups with 39,32 and 6 individuals inside each group. 
Table 10. Generalization capacity of different learning machines using all SNPs as input variables in CAU-RNASeq data set.

\begin{tabular}{|c|c|c|c|}
\hline Classification Mothod & Accuracy & AUC & Rolat.Abs. Error \\
\hline \hline NB & 56.38 & 0.67 & 72.83 \\
C4.5 & 68.71 & 0.77 & 53.73 \\
SVM & 67.95 & $\mathbf{0 . 8 1}$ & $\mathbf{5 3 . 5 3}$ \\
\hline Regrossion Mothod & RMSE & Corrolatton & Rolat. Abs. Error \\
\hline SVM-Reg. & 87.93 & 0.72 & 68.24 \\
Gaussian Proc. & 88.05 & 0.72 & 68.35 \\
Lasso & $\mathbf{7 7 . 9 6}$ & $\mathbf{0 . 7 9}$ & $\mathbf{6 0 . 7 6}$ \\
3-NN-reg-avg & 105.66 & 0.60 & 81.18 \\
5-NN-reg-avg & 100.62 & 0.63 & 76.85 \\
3-NN-reg-wgt & 105.81 & 0.60 & 81.34 \\
5-NN-reg-wgt & 100.62 & 0.63 & 76.90 \\
3NN-reg-krn & 111.80 & 0.57 & 86.78 \\
5NN-reg-krn & 113.00 & 0.55 & 84.94 \\
\hline
\end{tabular}

Table 11. Generalization capacity of different learning machines using all SNPs as input variables in YRI-RNASeq data set.

\begin{tabular}{|c|c|c|c|}
\hline Classification Mothod & Accuracy & AUC & Relat. Abs. Error \\
\hline \hline NB & $\mathbf{6 2 . 6 8}$ & 0.63 & 64.84 \\
C4.5 & 60.36 & 0.70 & 70.43 \\
SVM & $\mathbf{7 8 . 2 1}$ & 0.85 & $\mathbf{3 7 . 7 8}$ \\
\hline Rogrossion Mothod & RMSE & Corrolation & Relat. Abs. Error \\
\hline \hline SVM-Reg. & 93.63 & $\mathbf{0 . 6 4}$ & 79.84 \\
Gaussian Proc. & 93.69 & $\mathbf{0 . 6 4}$ & 79.93 \\
Lasso & 90.05 & 0.61 & 82.69 \\
3-NN-reg-avg & 98.05 & 0.59 & 81.17 \\
5-NN-reg-avg & 93.31 & 0.62 & 78.08 \\
3-NN-reg-wgt & 97.93 & 0.59 & 80.98 \\
5-NN-reg-wgt & 93.19 & 0.62 & $\mathbf{7 8 . 0 4}$ \\
3NN-reg-krn & 99.99 & 0.62 & 84.50 \\
5NN-reg-krn & 93.67 & 0.60 & 78.41 \\
\hline
\end{tabular}

\subsubsection{Block-Based Approach}

In this section we pursue the same analysis carried out in Section 3.1.3 for microarray data. In this case, among the classification algorithms we picked the SVM model because it discovered blocks with higher prediction capacity than C4.5 classifier. The Lasso regression model was chosen again because was one of the most competitive regressors. In Fig. (10), we display the results of this analysis. In Table 12 we detail the 10 blocks with the lowest relative absolute error for the classification and the regression model in the two populations. As it happened with the microarray data, classification algorithms performed better than regression algorithms because as made more accurate predictions with the key blocks associated with the RNASeq expression of DRB5. With these new data sets, we also sought whether the performance of a classification model using a set of SNPs was higher or not than the performance obtained using a single SNP. In that way, we compared AUC of the SVM classification model using all SNPs within the same block with respect to the AUC obtained using only one single SNP. We selected the SNP with the maximum AUC within the same block. In Fig. (11) we plot both measures for all the 345 blocks. In Table 13 we detail the 10 blocks with the highest AUC using the SVM classifier as well as the AUC obtained with the best performing single SNP among all SNPs within this block. In this case, it is curious to see how there were not the strong increments we observed with the same experiment using microarray data. In Section 4 we discuss this issue.

\section{DISCUSSION}

Results obtained for gene DRB5 when using single-SNP classifiers and microarrays have extensively been confirmed by common SNP-RNA expression correlation models. The levels of DR gene expression could condition the type of immune response. The high expression of DRB5 gene could increase the amount of DR receptor in the surfaces of the antigen presenting cell (APC) and as consequence increase 
(a) CAU RNASeq data

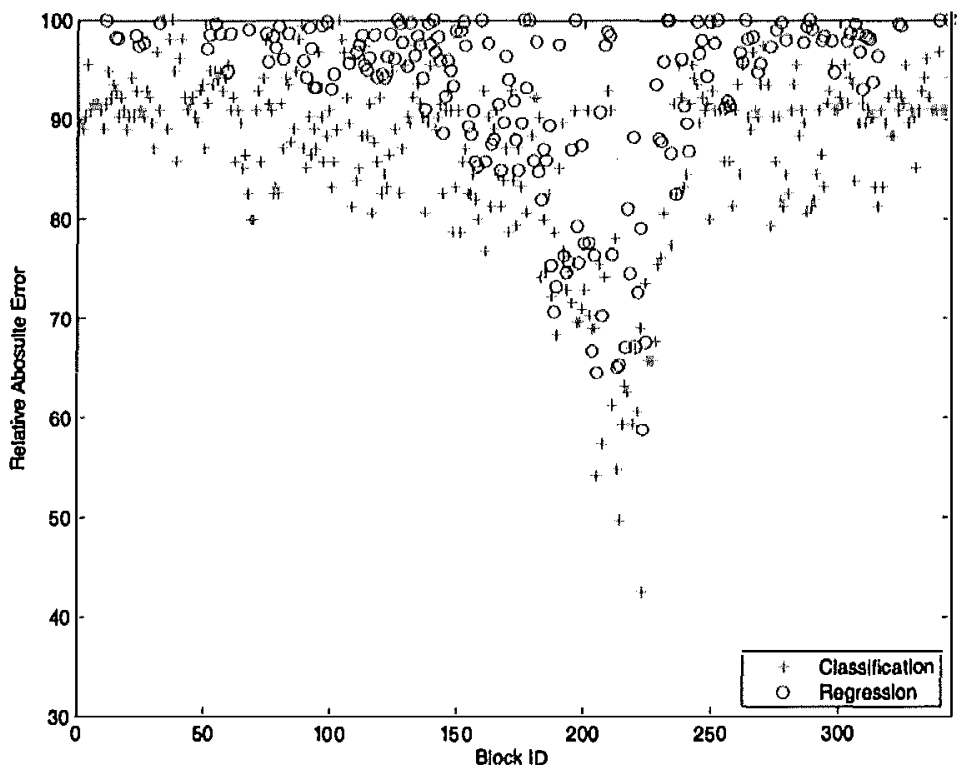

(b) YRI RNASeq data

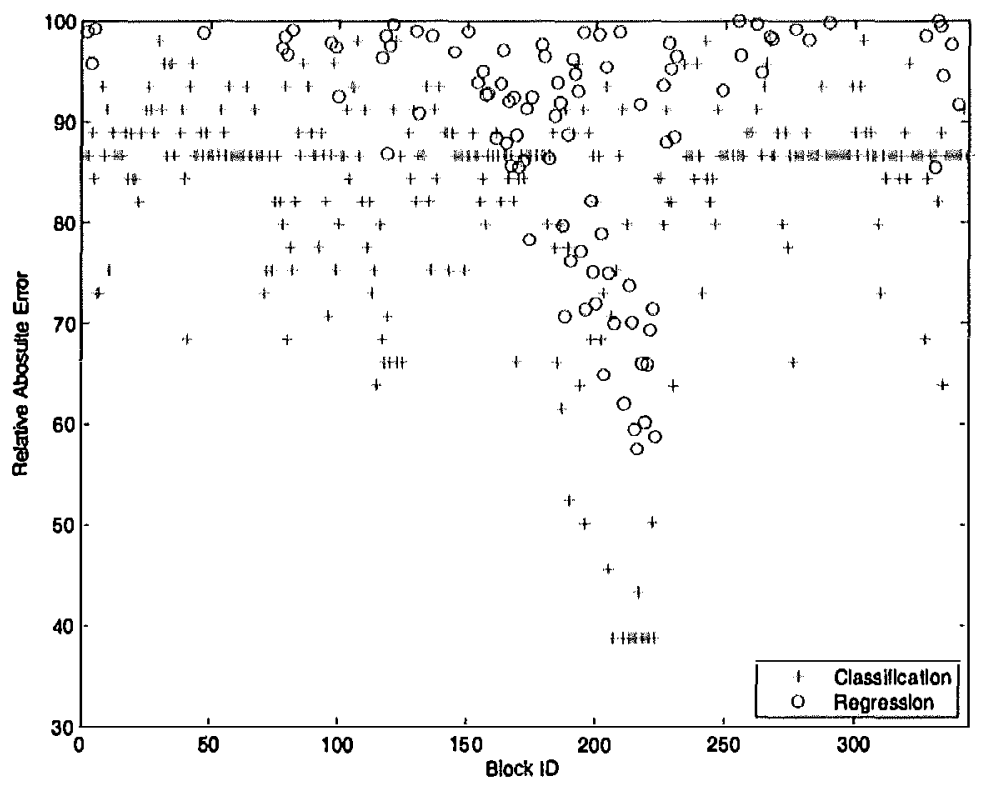

Fig. (10). Relative Absolute Error comparlson between a classificatlon model (SVM) and a regresslon model (Lasso). For the CAU-RNASeq population, the number of blocks wher e the classif Ication model obtalned a RAE $<50 \%$ was 2 and for RAE $<70 \%$ the number of blocks was 23. For the regression model in the CEU-array population, these numbers were 0 and 8 respectlvely. For the YRI-array population, the number of blocks where the classif Icatton model obtalned a RAE $<50 \%$ was 13 and for RAE $<70 \%$ the number of blocks was 34 . For the regression model in the YRI-array population, thesenumbers were 0 and 10 respectlvely.

the concentration of peptide-MHC complex and in turn affect the duration and specificity of the T cell-TCR with APC-HLA molecules interaction. The immunological synapse strength between APC and the T cell determines the fate of $T$ cells into Th1 or Th2 types [35] favoring Th1 differentlation when a stronger TCR signal is produced. 
Table 12. The 10 blocks with the lowest relative absolute orror (RAE) using a classification model (SVM) learnt with all SNPs within this block. 7 blocks (in bold) out of the 10 are shar ed by the two populations.

\begin{tabular}{|c|c|c|c|c|c|c|c|}
\hline \multicolumn{4}{|c|}{ CAU-RNASeq } & \multicolumn{4}{|c|}{ YRI-RNASeq } \\
\hline \multicolumn{2}{|c|}{ Discretized } & \multicolumn{2}{|c|}{ Continuous } & \multicolumn{2}{|c|}{ Discretized } & \multicolumn{2}{|c|}{ Continuous } \\
\hline Block ID & RAE & Block ID & RAE & Block ID & RAE & Block ID & RAE \\
\hline 223 & 42.57 & 223.00 & 58.88 & 207 & 38.75 & 216 & 57.47 \\
\hline 214 & 49.66 & 205.00 & 64.55 & 211 & 38.75 & 223 & 58.70 \\
\hline 205 & 54.18 & 213.00 & 65.08 & 213 & 38.75 & 215 & 59.44 \\
\hline 213 & 54.82 & 214,00 & 65.33 & 214 & 38.75 & 219 & 60.14 \\
\hline 207 & 57.40 & 203.00 & 66.76 & 215 & 38.75 & 211 & 62.00 \\
\hline 215 & 59.34 & 216.00 & 67.08 & 216 & 38.75 & 203 & 64.90 \\
\hline 219 & 59.34 & 220.00 & 67.20 & 218 & 38.75 & 220 & 65.93 \\
\hline 221 & 60.63 & 224.00 & 67.69 & 219 & 38.75 & 218 & 66.00 \\
\hline 211 & 61.27 & 207.00 & 70.27 & 220 & 38.75 & 221 & 69.27 \\
\hline 217 & 62.56 & 188.00 & 70.68 & 221 & 38.75 & 207 & 69,98 \\
\hline
\end{tabular}

Table 13. The 10 blocks with the highest AUC using a classification model (SVM). "Single SNP AUC" column displays the AUC obtained with the best performing single SNP among all SNPs within this block. 7 blocks (in bold) out of the 10 are shared by the two populations.

\begin{tabular}{|c|c|c|c|c|c|}
\hline \multicolumn{3}{|c|}{ CAU-RNASoq } & \multicolumn{3}{|c|}{ YRI-RNASoq } \\
\hline \hline Block ID & Block AUC & Single SNP AUC & Block ID & Block AUC & Singlo SNP AUC \\
\hline 223 & 0.771 & 0.797 & $\mathbf{2 0 7}$ & 0.789 & 0.788 \\
$\mathbf{2 1 4}$ & 0.733 & 0.695 & $\mathbf{2 1 1}$ & 0.789 & 0.776 \\
205 & 0.709 & 0.612 & $\mathbf{2 1 3}$ & 0.789 & 0.797 \\
$\mathbf{2 1 3}$ & 0.704 & 0.707 & $\mathbf{2 1 4}$ & 0.789 & 0.788 \\
$\mathbf{2 0 7}$ & 0.690 & 0.686 & $\mathbf{2 1 5}$ & 0.789 & 0.787 \\
$\mathbf{2 1 9}$ & 0.680 & 0.698 & 216 & 0.789 & 0.789 \\
$\mathbf{2 1 5}$ & 0.679 & 0.684 & 218 & 0.789 & 0.749 \\
$\mathbf{2 2 1}$ & 0.677 & 0.694 & $\mathbf{2 1 9}$ & 0.789 & 0.761 \\
$\mathbf{2 1 1}$ & 0.670 & 0.703 & 220 & 0.789 & 0.816 \\
217 & 0.662 & 0.654 & $\mathbf{2 2 1}$ & 0.789 & 0.772 \\
\hline
\end{tabular}

Therefore, the combination of DRB expression levels with specific structure receptors produced by the variants in the region would determine the fate of the T-cell ant the immune response [36].

However, in this work we have built multivariate models able to outperform single-model results, and have shown how by discretizing gene expression, classification learning machines can be used as an alternative tool to regression learning, which are robust to redundancy and noisy variables, when learning and testing complex models composed of hundred or thousand input variables. Moreover, some of them such as NB or C4.5 learn white-box models that can be interpretable by human experts. In the case of binarized gene expression, as in expression data sets from microarrays, NB classifler can be also understood as a Genetic Risk Score [37], a logistic regression widely used to predict individual predisposition to have a disease, considered as a binary trait, in which the output is interpreted as the probability of having or not the disease or, in our study, of having a high/low expression of gene DRB5.

Results obtained for gene DRB5 showed that there was always a classification approach that outperformed all regression models tried. Some multivariate models used in this study show their robustness to redundant variables, an important feature that will help model replication in an independent data set. In fact, those classification or regression learning algorithms able to perform variable filtering or weighting, i.e. C4.5 among classifters and Lasso among regression methods, showed higher performance in a cross-validation approach in the microarray data sets. By using all SNPs in the data sets most likely we are considering SNPs with no role in DRB5 expression that may introduce noise if not removed by the learning algorithms, or they may introduce redundancy if they are not causal but are in high LD with a causal SNP.

Multivariate models are very important whenever a single SNP may not completely explain the genetic effect on the expression level of a gene, either because the truly cause was not genotyped or because there is an epistatic effect among two or more causal loci. Our results conducted on microarray data showed how different blocks affected gene regulation and the multivariate models outperformed single SNP models in some of the blocks with best performance, showing agaln the importance of using robust multivariate 
(a) CAU RNASeq data

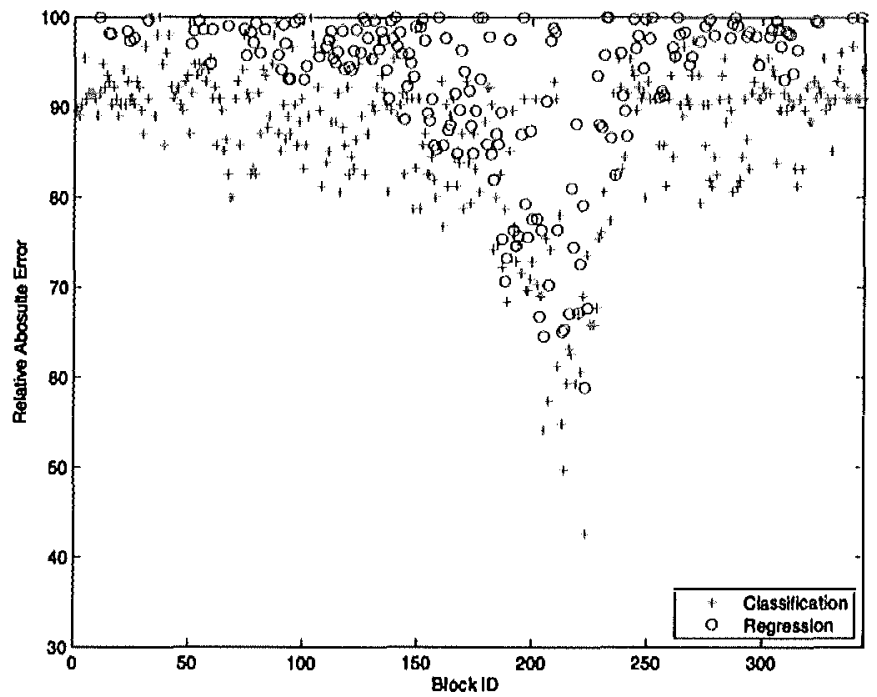

(b) YRI RNASeq data

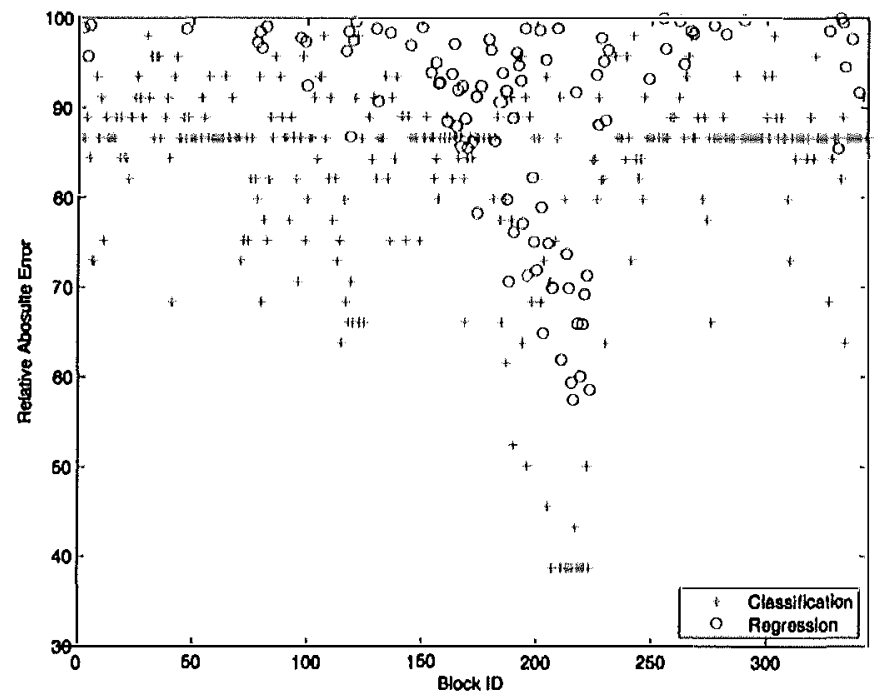

Fig. (11). AUC comparlson between a classffication model (SVM) using all SNPs in a blocki and the maximum performance obtalned using a single SNP withln the same block.

models. Results from RNASeq data set show the same pattern between classifiers and regression functions, with always a classifier outperforming all the regression models. However, they deserve a deep discussion as they, in all experiments and against the supposed superiority in power of RNASeq over microarray technology, underperformed those obtalned by microarray data.

The first important difference compared with microarrays was the distrlbution of expression values that showed a more complex pattern to be discretized. Perhaps sequencing errors explain thls complex pattern, which may also explain why predictlve capacity is never as high as the one reached by microarray data.

When comparing in an individual basis two-bins discretization from RNASeq with that from microarrays, by using only common individuals -1.e. data sets CEUcommonIndividuals and YRI-commonIndividuals- an interesting result arose: differences in discretization results between the two technologles (microarrays and RNASeq) depended on the population. Therefore, YRI-RNASeq could be up-blased, as there were 18 individuals with different discretlzed expresslon level (low or high) between the two technologles but all of them had expression levels always 
classifled as low in YRI-array and as high in YRI-RNASeq On the contrary, almost all individuals 14 out of 18 with different expression level (low or high) between the two technologles had expresslon levels always classif ied as high in CEU-array and as low in CEU-RNASeq. If we assume that DRB5 expression from arrays was correctly discretized because Its simplicity (see Fig, 1) and the perfect classification accuracy when estimation from genotypes reached by some classifiers, we may have found an interesting situation in which the theoretically more accurate RNASeq brought worse results and the blas depended on the population, so that CEU individuals tended to have lower expression of DRB5 and YRI tended to have higher expression of DRB5. Some issues in a still very novel NGS technique have been suggested before [32]. Moreover, predictive accuracy behaved very differently between populations in whole, block-based and single models. As an example, in whole models differences between microarray and RNASeq were large in Caucasian: SVM and C4.5 had same accuracy in Caucasian and much lower than with microarray data (69.5 in CAU-RNASeq versus 100 and 90,65 in CEU-RNASeq for C4.5 and SVM respectively). However, when using YRI data differences were outstanding: SVM clearly outperformed C4.5 (77.92 versus 40.26) in RNASeq when in microarrays It was clearly worse (79.5 versus 99). In presence of large amounts of errors in RNA sequencing, varlable selectlon may not be that good, as the errors show up more clearly when only a few varlables are selected than whenever all varlables are used, given that the whole DNA reglon was selected to be close to where DRB5 gene is encoded. Some possible explanation of the lack of accuracy in RNASeq data sets is that genes may have several lsoforms, many of them sharing exons so that some reads cannot be unequivocally assigned to a transcript [38]. This effect may even occur between genes, as among DRB1 and DRB5 genes. In our situation, even If DRB5 has several transcripts, only one was used when mapping reads to genes. It could be that other transcripts common in CEU and performing the same molecular effect were expressed but not captured. At the same time, it is already known that some reads map exons in both DRB5 and DRB1 gene. As only one Isoform was used for DRB5, it could exist an isoform common in YRI very similar to the one used in the readmapping phase, which caused the up-blas in YRI-RNASeq.

\section{CONCLUSION}

We have shown how several classification algorithms, which are robust to redundant and nolse varlables, show a high predictive accuracy. Some of the classification approaches have revealed to be very helpful for blomedical researchers, as they have learned white-box models easily interpretable by human experts.

Feature selection of SNPS based on LD criterion has helped to Identify SNPs that may be candidate eQTLs in the predictive models. Because of their low computational complexity to the number of input variables, we have been able to use very robust classification algorlthms under different approaches with all the SNPs within the vicinity of genes DRB1 and DRB5.

These conclusions are more difficult to obtain when using regression models, as the larger complexity of a regression model compared with a classifier translated into a reduction in robustness to redundancy and therefore in generalization capacity and interpretability. However, when they were able to perform varlable filtering some way, such as in Lasso regression they performed much better.

Finally, although these results have been replicated with RNASeq data, they show very different and unexpected patterns, such as a lower performance compared with microarrays perhaps because a high level of noise introduced in the sequencing process, which is also blased depending on the population. This high degree of sequencing errors may explain a better performance reached by models that did not perform varlable selection, All these results, discussed above, may be revealing several open issues in a very novel NGS technology and will need deeper research.

Glven these results, one of our maln short-term challenges is to determine the functional link between the SNPs and the phenotype at two levels, DRB5 expression and disease Implication. We plan to focus on Copy Number Varlations (CNV), since there are multiples CNV in the HLA region. We will try to connect the SNPs discovered in this work with insertions or deletions of the DRB5 gene. On the other hand, ENCODE database clusters information about genome-wide regulatory reglons. This information can be cross with the data obtalned in the present work with the objectlve to determine which is the ultimate cause of the DRB5 expression levels.

\section{ABBREVIATIONS}

$$
\begin{array}{ll}
\text { AUC }= & \text { Area Under the ROC Curve } \\
\mathrm{cV}= & \text { Cross Validation } \\
\mathrm{CEU}= & \begin{array}{l}
\text { Samples of Utah residents with Ancestry from } \\
\text { Northern and Western Europe Used by }
\end{array} \\
& \text { HapMap and } 1000 \text { Genome Projects } \\
\text { NGS }= & \text { Next Generation Sequencing } \\
\text { RNASeq = } & \text { RNA Sequencing Technology } \\
\text { SNP }= & \text { Single Nucleotide Polymorphism } \\
\text { YRI }= & \text { Samples of Yoruba from Ibadan, Nigerla Used } \\
& \text { by HapMap and } 1000 \text { Genome Projects }
\end{array}
$$

\section{CONFLICT OF INTEREST}

The authors declare that they have no competing interests.

\section{ACKNOWLEDGEMENTS}

The authors were supported by the second call program for research and development of the International Excelence Campus BioTic Granada under microproject CEI-mic20132, and under research project CEI-ID1-2013-15, the Spanish Research Program under projects TIN2010-20900-C04-1 and TIN2013-46638-C3-2-P, the Andalusian Research Program under project P08-TIC-03717 and the European Reglonal Development Fund (ERDF), RA is currently supported by NIH grant 1 R01NS0860832 under the CRCNS program. 


\section{REFERENCES}

[I] Wallace C, Rotival M, Cooper JD, et al. Statistical colocalization of monocyte gene expression and genetlc risk varlants for type 1 diabetes. Hum Mol Genet 2012; 21(12): 2815-24.

[2] Gat Viks I, Meller R, Kuplec M, Shamlr R. Under standing gene sequence varlation in the context of transcription regulation in yeast. PLoS Genet 2010; 6(1): e1000800.

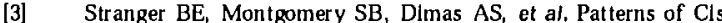
Regulatory Varlation in Diverse Human Populations. PLoS Genet 2012; 8(4): el 002639

[4] Llu H, LI J, Wong L. A Comparative Study on Feature Selection and Classification Methods Using Gene Expression Profiles and Proteoml c Patterns. Genome Inform 2002; 13: 51-60,

[5] Ross ME, Zhou $X$, Song $G$, et al, Classification of pedlatrlc acute lymphoblastic leukemla by gene expression profillng. Blood 2003: 102(8): 2951-9

[6] Wanga $Y$, Tetko IV, Hall MA, et as. Gene selection from microarray data for cancer classificatlon, a machine learning approach. Comput Blol Chem 2004; 29(1): 37-46.

[7] Abad-Grau M, Medina-Medina N, Montes-Soldado R, Matesanz F, Bafna V. Sam ple Reproduclbillty of Genetlc Association using DIff erent Multimarker TDTs in Genome wide Association Studles: Characterization and a New Approach. PLoS ONE 2012; 7(2): 29613.

[8] Fogdell A, Hillert J, Sachs C, Olerup O. The multiple sclerosis and narcolepsy assoclated HLA class II haplotype includes the DRB5*0101 allele. Tissue Antlgens 1995; 46: 333-6.

[9] VincentR, PPL, GongoraC, Papal, et al. JCJ. Quantltative analysis of the expresslon of the HLA-DRB genes at the transcriptlonal level by competitive polymerase chaln reaction. J Immunol 1996; 156: 603-10.

[10] Schadt EE, Molony C, Chudin E, et al. XY, Mapping the genetlc architecture of gene expression in human Ilver. PLoS Blology 2008; 2008: 6: e 107 .

[11] Dixon AL, Llang L, Moffatt MF, Chen W, et al. SH, A genomewide assoclation study of global gene expresslon. Nat Genet 2007 39: $1202-7$.

[12] JA JQ, Huh J, M MB, et al. Myelin basic proteln-speciflc TCR/HLA-DRB $5^{*} 01$ : 01 transgenic mice support the etlologic role of DRB5*01: 01 In multiple sclerosis. J Jmmuno 2012; 189(6): 2897-908.

[13] Pearl J. Probabilistic Reasoning with Intelligent Systems. San Mateo: Morgan \& Kaufman: 1988

[14] Dempster AP, Laird NM, Rubln DB. Maximum likelihood from incomplete data via the EM algorlthm. J Roy Stat Soc B Met 1977; 39 (1): 1-38.

[15] EverittBS. A finite mixture model for the clustering of mlxed mode data. Stat Probabil Lett 1988; 6(5): 305-9

[16] Duda RO, Hart PE. Pattern Classification and Scene Analysis. New York: John Wiley Sons, 1973.

[17] Fawcett T. An introduction to ROC analysis. Pattern Recogn Lett 2006; 27(8): $861-74$

[18] Quinlan JR. C4.5: Programs for Machine Learning. San Francisco: Morgan Kaufmann; 1993

[19] Hall M, Frank E, Holmes G, Pfahringer B, Reutemann P, Witten IH. The WEKA Data Mining Software: An Update. In: SICKDD Explorations 2009; 11: 1 .
[20] Chang CC, Lin CJ. LIBSVM: A library for support vector machlnes. ACM Trans Intel Syst Technol 2011; 2: 1-27. Sof tware avallable at http: //www.csle.ntu.edu.tw/ cjlin/libsvm

[21] Shevade SK, Keerthi SS, Bhattacharyya $C$, Murthy KRK. Improvements to the SMO algorlthm for SVM regression. IEEE Trans Neural Networks Learning Syst 2000; 11 (5): 1188-93.

[22] Williams C, Rasmussen C. Gaussian Processes for Regr ession. In: Advances in Neural Information Processing Systems 1996; 8: 51420.

[23] Tibshirani R. Regression shrinkage and selection via the Lasso. J Roy Stat Soc B 1996; 67(1): 91-108.

[24] Cover $T$, Estimation by the nearest neighbor rule. IEEE Transactions on Information Theory 1968; 14(1): 50-5.

[25] Hammlng RW, Error detecting and error correcting codes. Bell System Technical Journal 1950; 29(2): 147-60.

[26] Gabriel $S$, Schaffner $S$, Nguyen $H_{1}$ et at. The Structure of Haplotype Blocks in the Human Genome. Sclence 2002; 296: $2225-9$.

[27] Hap Map Consortlum TI, The International Hap Map Project. Nature 2003; 426: 789-796.

[28] Hap Map Consortlum TI. Integrating common and rare genetlc varlation in diverse human populatlons. Nature 2010: 467(7311): 52-58. A vallable from: http: //dx,dol.org/10.1038/nature09298.

[29] Howle BN, Donnelly P, Marchinl J. A flexlble and accurate genotype Imputation method for the next generation of genome wlde association studies. PLoS Genetics 2009; 5(6); el 000529.

[30] Dermitzakis E. E-GEUV-1 - RNA-sequencing of 465 lymphoblastold cell lines from the 1000 Genomes; 2013. http: //www.ebi.ac,uk/arrayexpress/experiments/E-GEUV-1/.

[31] Consortlum GP, Abecasis G, Altshuler D, et al. A map of human genome varlation from population scale sequen cing. Nature 2010 467(7319): 1061-1073. Avallable from: http: //dx.dol.org/10.1038/ nature 09534 ,

[32] Trapnell $C$, Roberts $A$, Goff $L$, et al. Differentlal gene and Trapnell $C_{1}$ Roberts $A_{1}$, Gorf $L_{\text {, et al. Differential gene and }}$ Hat and Cuffinks. Nat Protocols 2012: 7(3): 562-578. A vallabl from: http: //dx.dol, or g/10.1038/nprot.2012.016.

[33] Kohavi R. A Study of Cross. Validation and Bootstrap for Accuracy Estimation and Model Selection. In: Proceedings of the 15th International Joint Conference on Artificial Intelligence 1995; 114 119.

[34] Fraley C, Raftery AE, How many clusters? Which clustering method? Answers via model-based cluster analysis. The Comp 1998; 41(8): 578-588

[35] Corse E, RA RAG, Allison JP. Strength of TCR-peptide/MHC interactions and in vivo $\mathrm{T}$ cell responses. J Immuno 2011; 186 5039-45.

[36] Alcina A, Abad-Grau MDM, Fedetz $M$, et al. Multiple sclerosis risk varlant HLA-DRB1*1501 assoclates with high expression of DRB1 gene In diff erent human populations. PLoS One 2012; 7(1): e29819.

[37] Sebastiani P, Solovieff N, Sun JX. Nave Bayeslan classifier and genetlc risk score for genetlc risk predictlon of a categorlcal tralt: not so different after all. Front Genet 2012; 3(26): do 10.3389/fgene.2012.00026.

[38] Garber M, Grabherr MG, Guttman M. Trapnell C. Computational methods for transcriptome annotation and quantification using RNA-seq. Nat Methods 2011; 8(6): 469-477. Available from: http: $/ / \mathrm{dx}$.doi,org/10.1038/nmeth. 1613 . 